Publications of the Astronomical Society of the Pacific, 115:362-382, 2003 March

(C) 2003. The Astronomical Society of the Pacific. All rights reserved. Printed in U.S.A.

\title{
SpeX: A Medium-Resolution 0.8-5.5 Micron Spectrograph and Imager for the NASA Infrared Telescope Facility
}

\author{
J. T. Rayner, ${ }^{1}$ D. W. Toomey, P. M. Onaka, A. J. Denault, W. E. Stahlberger, W. D. Vacca, ${ }^{1,2}$ And M. C. Cushing ${ }^{1}$ \\ Institute for Astronomy, University of Hawaii, 2680 Woodlawn Drive, Honolulu, HI 96822; rayner@.ifa.hawaii.edu \\ AND \\ S. WANG ${ }^{3}$
}

University of Chicago Engineering Center, 5640 South Ellis Avenue, Chicago, IL 60637

Received 2002 August 31; accepted 2002 November 2

\begin{abstract}
We present the design, construction, and performance of SpeX, a medium-resolution 0.8-5.5 $\mu \mathrm{m}$ cryogenic spectrograph and imager, now in operation at the $3.0 \mathrm{~m}$ NASA Infrared Telescope Facility (IRTF) on Mauna Kea. The design uses prism cross-dispersers and gratings to provide resolving powers up to $R \sim 2000$ simultaneously across $0.8-2.4,1.9-4.2$, or $2.4-5.5 \mu \mathrm{m}$, with a $15^{\prime \prime}$ long slit. A high-throughput low-resolution $R \sim 200$ prism mode is also provided for faint-object and occultation spectroscopy. Single-order $60^{\prime \prime}$ long-slit modes with resolving powers up to $R \sim 2000$ are available for extended objects. The spectrograph employs an Aladdin $31024 \times 1024 \mathrm{InSb}$ array and uses narrow slits and a spatial scale of 0.15 pixel $^{-1}$ for optimum sensitivity on point sources. An autonomous infrared slit viewer is used for object acquisition, infrared guiding, and scientific imaging in the wavelength range $0.8-5.5 \mu \mathrm{m}$. The imager employs an Aladdin $2512 \times 512 \mathrm{InSb}$ array that covers a $60^{\prime \prime} \times 60^{\prime \prime}$ field of view at $0.12 \mathrm{pixel}^{-1}$. SpeX was successfully commissioned on IRTF during 2000 May, June, and July. Astronomical observations are presented to illustrate performance.
\end{abstract}

\section{INTRODUCTION}

SpeX is a medium-resolution $0.8-5.5 \mu \mathrm{m}$ cryogenic spectrograph designed and built at the Institute for Astronomy, University of Hawaii, for the NASA Infrared Telescope Facility (IRTF) on Mauna Kea. The IRTF is a $3.0 \mathrm{~m}$ telescope owned by NASA and operated by the Institute for Astronomy, University of Hawaii, under contract to NASA. It is fully accessible to the national and international scientific community. Fifty percent of the observing time is reserved for solar-system objects, and the other $50 \%$ is available for other astronomical studies.

The $\sim 1-5 \mu \mathrm{m}$ region of the infrared spectrum is particularly well suited to studies of astrophysically cool objects $(T<$ $2000 \mathrm{~K}$ ), sources heavily obscured by dust, and objects at very high redshift. This wavelength region includes key diagnostic spectral features, notably the vibration-rotation bands of astrophysically important molecules (such as $\mathrm{CO}, \mathrm{OH}, \mathrm{SiO}, \mathrm{CH}$, $\mathrm{CN}, \mathrm{NH}, \mathrm{H}_{2} \mathrm{O}, \mathrm{HCN}, \mathrm{CH}_{4}$, etc.), the pure rotation transitions of $\mathrm{H}_{2}$, many atomic and ionic transitions, and many solid-state bands that are crucial diagnostics of the composition of dust,

\footnotetext{
${ }^{1}$ Visiting Astronomer at the Infrared Telescope Facility, which is operated by the University of Hawaii under contract from NASA.

${ }^{2}$ Currently at the Max-Planck-Institut für extraterrestrische Physik, Giessenbachstrasse, Garching, Germany.

${ }^{3}$ Currently at the CMC Flight Visions, 43W752 Route 30, P.O. Box 250, Sugar Grove, IL 60554.
}

ices, and the surfaces of solid bodies. The current complement of IRTF facility instruments includes NSFCAM (Shure et al. 1994), a 1-5.5 $\mu \mathrm{m}$ camera, and CSHELL (Tokunaga, Toomey, \& Carr 1990; Greene et al. 1993), a 1-5.5 $\mu \mathrm{m}$ high-resolution $(R=5000-40,000)$ spectrograph. SpeX fills the gap in spectral resolving power by providing low- and medium-resolution spectroscopy $(R=50-2500)$ across the range $0.8-5.5 \mu \mathrm{m}$.

\section{CHOICE OF SPECTRAL FORMATS}

The guiding design principle of SpeX was to optimize observing efficiency by maximizing simultaneous wavelength coverage using cross-dispersion. The spectral format was designed to fit the $0.8-5.5 \mu \mathrm{m} 1024 \times 1024$ Aladdin array. At spectral resolving powers of a few thousand, the background in the $0.8-2.5 \mu \mathrm{m}$ regime is dominated by $\mathrm{OH}$ sky-line emission. In contrast, thermal background emission from the telescope and sky rises by a factor of $10^{5}$ times from 2.5 to $5.5 \mu \mathrm{m}$. Therefore, it is best to cross-disperse and optimize the instrument for these two regions separately. The spectrograph modes are detailed in Table 1 and illustrated in Figures 1, 2, and 3.

\subsection{Advantages of Cross-Dispersion over Single-Order Spectroscopy}

Cross-dispersion provides three distinct advantages over single-order spectroscopy: 
TABLE 1

SPECTROGRAPH Modes

\begin{tabular}{|c|c|c|c|c|}
\hline Mode & $\begin{array}{c}\lambda \\
\text { Range } \\
(\mu \mathrm{m})\end{array}$ & $R^{\mathrm{a}}$ & $\begin{array}{l}\text { Slit Length } \\
\text { (arcsec) }\end{array}$ & Order Sorter \\
\hline SXD . & $0.8-2.4$ & 2000 & 15.0 & FS/ZnSe prism \\
\hline LXD1.9 & $1.9-4.2$ & 2500 & 15.0 & $\mathrm{LiF} / \mathrm{Ge}$ prism \\
\hline LXD2.1 & $2.1-5.0$ & 2500 & 15.0 & $\mathrm{LiF} / \mathrm{Ge}$ prism \\
\hline LXD2.3 & $2.3-5.4$ & 2500 & 15.0 & $\mathrm{LiF} / \mathrm{Ge}$ prism \\
\hline Low-resolution (prism) & $0.8-2.5$ & 250 & 15.0 or 60.0 & Not required \\
\hline Short single order $\ldots .$. & $0.8-2.5$ & 2000 & 15.0 or 60.0 & Filters ${ }^{\mathrm{b}}$ \\
\hline Long single order . & $1.9-5.5$ & 2500 & 15.0 or 60.0 & Filters $^{\mathrm{c}}$ \\
\hline
\end{tabular}

${ }^{\text {a }}$ Average spectral resolving power matched to a $0.3 \mathrm{slit} ; R$ is proportionately lower with wider slits. Available slit widths: 0"3, 0"5, 0"8, 1".6, and 3".0.

${ }^{\mathrm{b}}$ Available order-sorting filters: $0.91-1.00,1.03-1.17,1.17-1.37,1.47-1.80$, and $1.92-2.52 \mu \mathrm{m}$.

${ }^{c}$ Available order-sorting filters: $3.13-3.53,3.59-4.41$, and 4.40-6.00 $\mu \mathrm{m}$.

1. Efficient use of telescope time.-SpeX can acquire the entire 0.8-5.5 $\mu \mathrm{m}$ region in just two instrument settings. Covering this range in single-order mode would require 10 different settings at $R \sim 2000$ and would be compromised by lack of overlap between orders. The two cross-dispersed modes overlap at $2.4 \mu \mathrm{m}$ to allow relative calibration of the two spectra. It is the reduced number of instrument settings that significantly improves efficiency.

2. Simultaneous wide spectral coverage.-Simultaneous observations guarantee that spectra are taken at the same time and come from the same position. This feature removes the uncertainty introduced by using sequential observations since variations in seeing and guiding can introduce normalization problems when piecing together spectra not taken simultaneously. Cross-dispersion is therefore optimum for gathering

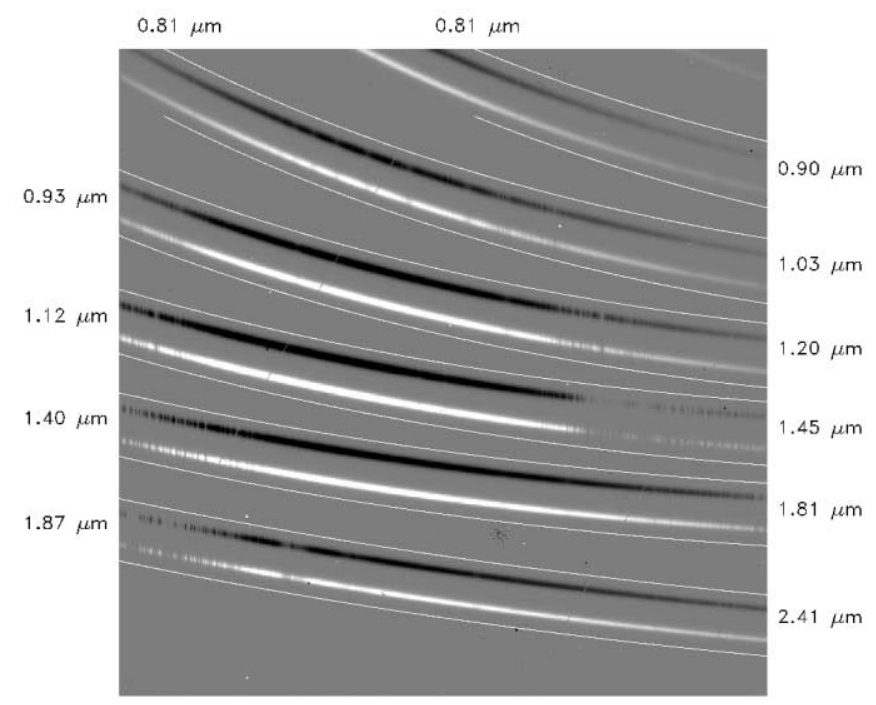

FIG. 1.-SXD mode. Short-wavelength $(0.8-2.4 \mu \mathrm{m})$ cross-dispersed format. Shown is an object minus sky image where a star has been nodded 7".5 in the $15{ }^{\prime \prime} .0$ slit. The white lines indicate the top and bottom of the slit. Note the lowtransmission telluric features.

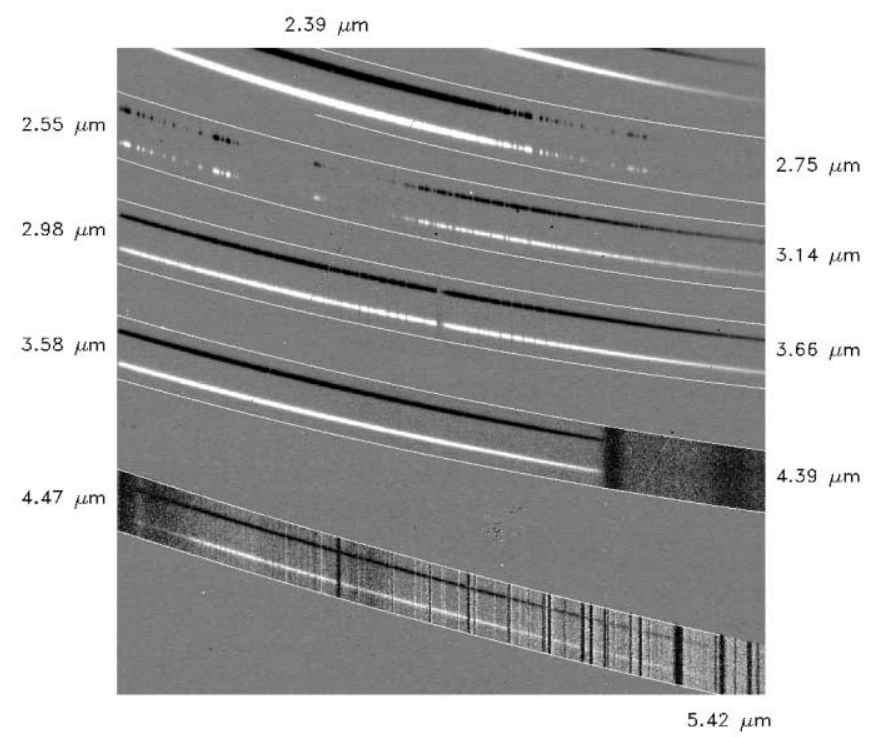

FIG. 2.-LXD2.3 mode. Long-wavelength (2.3-5.4 $\mu \mathrm{m})$ cross-dispersed format. Shown is an object minus sky image where a star has been nodded 7".5 in the $15 " 0$ slit. At thermal wavelengths, telluric features emit. Depending on sky background stability, this can result in positive (white) or negative (black) telluric features in the spectrum.

widely separated lines and calculating precise line ratios. It is also indispensable for high time resolution on variable objects such as comets, asteroids, planets, and variable stars. Additional efficiencies accrue from providing relatively calibrated spectra under marginal weather conditions.

3. High throughput.-Order-sorting interference filters are not required since order sorting is done by the prisms, and

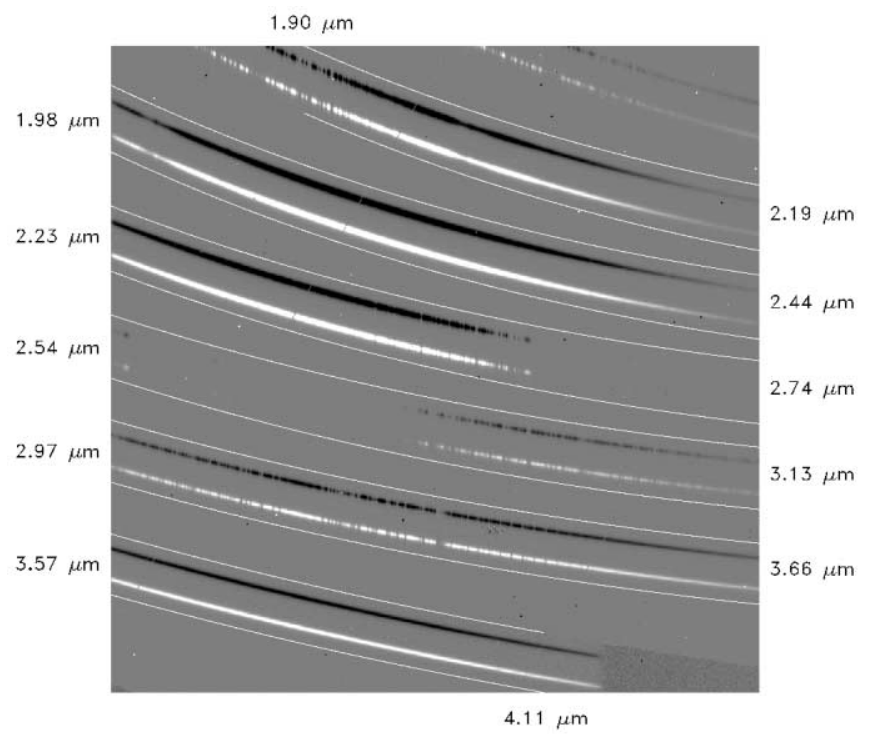

FIG. 3.-LXD1.9 mode. Long-wavelength (1.9-4.2 $\mu \mathrm{m})$ cross-dispersed format. Same as Fig. 2, except that the $K, L$, and $L^{\prime}$ bands are covered simultaneously by rotating the grating turret by 0.95 . 
prisms have higher transmissions than interference filters. Cross-dispersion is also much more efficient at the wavelength of overlapping orders where order-sorting filters must have high attenuation to avoid interference from the other orders. Also, since cross-dispersion sorts orders spatially, wavelengths at the edge of each order occur at two displaced positions, and signals from these two positions can be combined to compensate for the loss of efficiency that results from working away from the blaze angle at the center of each order.

\subsection{8-2.4 $\mu \mathrm{m}$ Cross-dispersed (SXD) Mode}

Without adaptive optics, the very best image quality obtainable on the IRTF is about 0".3 FWHM at $2.2 \mu \mathrm{m}$. Hence, the pixel size in the spectrograph is set to 0.15 for Nyquist sampling. The cross-dispersed slit length is set to $15^{\prime \prime}$ (100 pixels). This is sufficient to allow telescope nodding of point sources within the slit, eliminating the need for separate sky positions.

With a suitable choice of cross-dispersing prism materials ( $\mathrm{ZnSe}$ and fused silica), it has been possible to keep the spacing between orders almost constant, even though SpeX works in low orders to ensure maximum wavelength coverage. This results in a very efficient use of the array format.

With a slit length of 100 pixels $\left(15^{\prime \prime}\right)$, about six orders comfortably fit across 1024 pixels, allowing for tilt and curvature of individual orders and including some interorder spacing to enable vertical repositioning to avoid possible bad-pixel clusters on the array. To match the center of each order with the most transparent parts of the atmosphere, the standard approach of placing $2.2 \mu \mathrm{m}$ in third order and $1.65 \mu \mathrm{m}$ in fourth order, etc., is taken. The blaze (i.e., central) wavelength $\lambda_{m}$ in order $m$ is given by

$$
\lambda_{m}=\frac{\lambda_{1}}{m},
$$

where the blaze wavelength in first order $\lambda_{1}=6.6 \mu \mathrm{m}$ in this case. The long- $\left(\lambda_{l}\right)$ and short-wavelength $\left(\lambda_{s}\right)$ limits (theoretically $40 \%$ of the blaze intensity, although in practice the intensity is less) of each order are given by (Schroeder 2000):

$$
\begin{aligned}
& \lambda_{l}=\frac{m \lambda_{m}}{m-1 / 2}, \\
& \lambda_{s}=\frac{m \lambda_{m}}{m+1 / 2} .
\end{aligned}
$$

With the 2 pixel $(0.3$ slit) resolving power set to $R=2000$ $\left(120 \mathrm{~km} \mathrm{~s}^{-1}\right)$, third order spans the $K$-band and orders 3-8 cover $2.4-0.8 \mu \mathrm{m}$ as desired (see Fig. 1), but with a small gap in wavelength coverage at $1.81-1.86 \mu \mathrm{m}$ in the deep telluric feature. No-gap coverage across this range would have meant reducing the resolving power to $R=1500$. A resolving power of $R=2000$ adequately separates $\mathrm{OH}$ emission lines (roughly
$30 \%$ of the pixels from 0.8 to $2.5 \mu \mathrm{m}$ are covered by $\mathrm{OH}$ lines of varying intensity).

At a resolving power of $R=2000$ on a $3.0 \mathrm{~m}$ telescope such as IRTF, the limiting sensitivity depends strongly on array performance. The sky background between the $\mathrm{OH}$ lines at the detector is about $0.015 e^{-} \mathrm{s}^{-1}$ pixel $^{-1}$ (for an instrument throughput of $15 \%$ and 590 photons $\mathrm{s}^{-1} \mathrm{~m}^{-2} \operatorname{arcsec}^{-2} \mu \mathrm{m}^{-1}$ from the sky at $1.65 \mu \mathrm{m}$; Maihara et al. 1993), which is well below the measured dark current of $0.2 e^{-} \mathrm{s}^{-1}$. The measured read noise is $12 e^{-}$rms with Fowler multiple sampling (Fowler $\&$ Gatley 1990), and so integration times of longer than 20 minutes are required to become dark current limited. (In practice, integration time is limited by residual image effects. See $\S 8.1$ for further discussion.)

\subsection{9-5.4 $\mu \mathrm{m}$ Cross-dispersed (LXD) Mode}

As was the case with the $0.8-2.4 \mu \mathrm{m}$ cross-dispersed mode, a suitable choice of prism materials ( $\mathrm{Ge}$ and $\mathrm{LiF}$ in this case) keeps the spacing between orders about constant. The slit length is also $15^{\prime \prime}$, and up to six orders can be covered simultaneously. Unlike the $0.8-2.4 \mu \mathrm{m}$ mode, however, there is no natural way of matching the center of each order with the most transparent parts of the atmosphere. Since the background is too large to obtain useful spectroscopy on many objects in the $M$ band $(4.5-5.4 \mu \mathrm{m})$, the spectral format has been arranged so that the $M$ band spans order 4 and so that the $L$ and $K$ bands occupy orders 5-10. This allows the $M$ band to be moved off the array if desired. Depending on the chosen fine position of the grating turret, orders 4-8 (5.4-2.3 $\mu \mathrm{m}$; see Fig. 2) or orders 5-10 (4.1-1.9 $\mu \mathrm{m}$; see Fig. 3) fit onto the array at a spectral resolving power of $R=2500$ matched to a 0.3 slit, while again leaving space to avoid possible bad areas of the array. Sky brightness limits on-chip exposure times to about $30 \mathrm{~s}$ at $4 \mu \mathrm{m}$ and to about $2 \mathrm{~s}$ at $5 \mu \mathrm{m}$. Consequently, if integration times are set by the sky background, the LXD mode is read noise limited at wavelengths shorter than about $3 \mu \mathrm{m}$.

\subsection{8-2.5 $\mu \mathrm{m}$ Low-Resolution Prism Mode}

This mode is designed for occultations and faint-object spectroscopy. The $0.8-2.5 \mu \mathrm{m}$ region is dispersed by a single prism onto one quadrant of the $1024 \times 1024$ array for fast readout. Since no gratings are required and the spectral resolving power is low (average $R=250$ matched to a 0.3 slit), spectra can be acquired quickly and efficiently. In the prism mode, angular dispersion is a function of refractive index, and resolving power varies by about a factor of 2 from $0.8 \mu \mathrm{m}\left(R=150 / 0^{\prime \prime} .3\right.$ slit $)$ to $2.5 \mu \mathrm{m}\left(R=350 / 0^{\prime \prime} .3 \mathrm{slit}\right)$. The prism mode also overcomes some shortcomings of the $0.8-2.4 \mu \mathrm{m}$ cross-dispersed (SXD) mode. The slit length is not restricted to $15^{\prime \prime}$, and a $60^{\prime \prime}$ slit can be used (although there is some curvature for slits this long). The throughput of the prism mode is high and relatively constant (see $\S 4.6$ ) across $0.8-2.5 \mu \mathrm{m}$. By comparison, the SXD mode has lower average throughput because gratings are less 


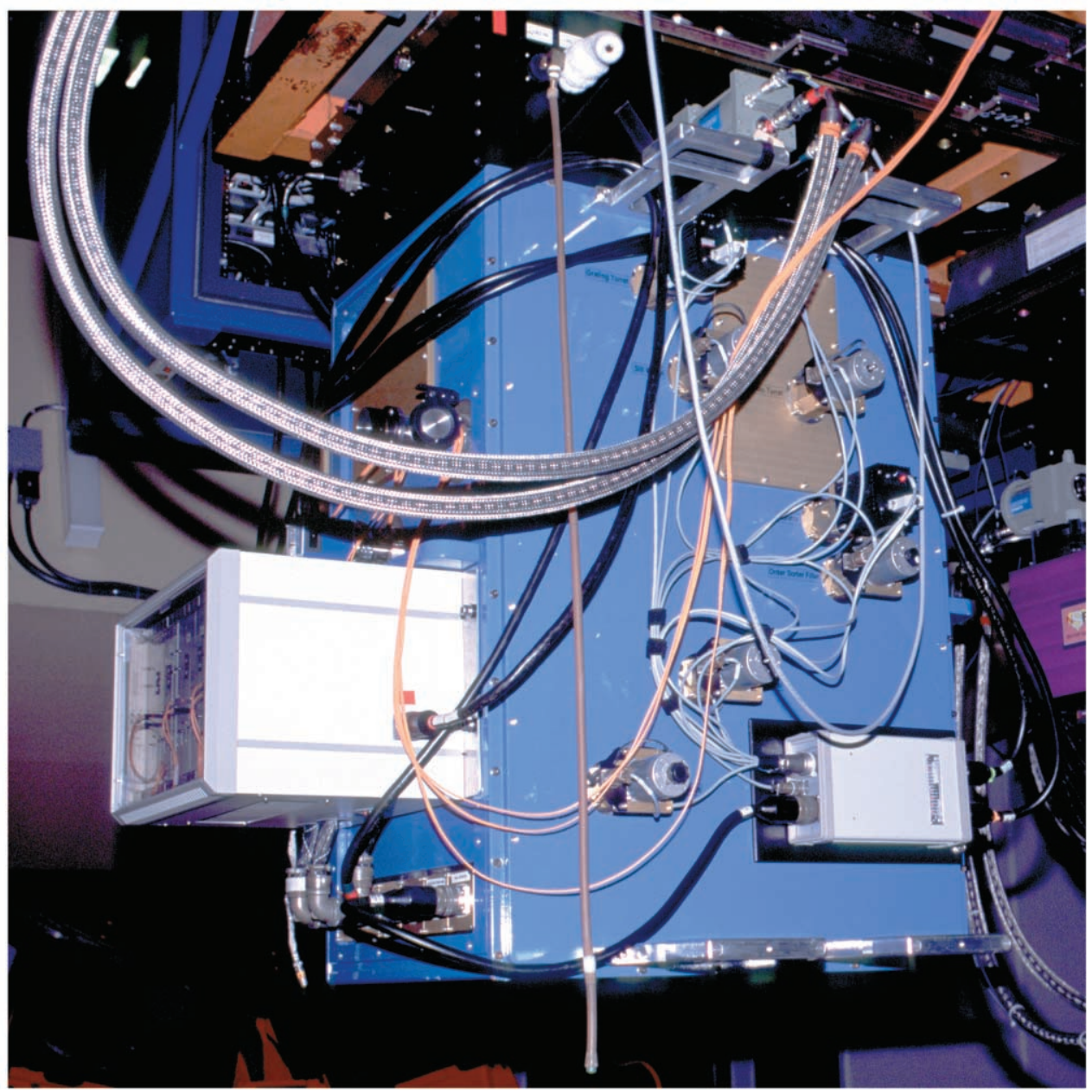

FIG. 4.-Photograph of SpeX on IRTF. The cryostat (blue box) is mounted to the telescope by the black interface box, which contains the calibration unit. The closed-cycle cooler is visible at the top of the cryostat. Visible on the front cover of the cryostat are motors for controlling the seven cold mechanisms. Mounted to the side of the cryostat at the lower left is the cryostat-mounted electronics box containing clock, bias preamp, analog-to-digital converter, and fiber-optic boards. For scale, SpeX is $1.4 \mathrm{~m}$ tall and weighs $478 \mathrm{~kg}$.

efficient. SXD efficiency is also reduced at the edges of each order because of the grating blaze function. The average throughput of the prism mode is about twice that of the SXD mode.

\subsection{Single-Order Long-Slit Modes}

The long-slit modes add a further degree of instrument flexibility on extended objects such as planets, comets, nebulae, and galaxies, even though the small pixel size $\left(0.15\right.$ pixel $\left.^{-1}\right)$ is not optimal for low surface brightness objects. To keep optical components within reasonable limits, the longest slit is 60 (400 pixels). The two single-order long-slit modes are identical to the SXD and LXD modes, except the gratings are not paired with a cross-dispersing prism, and order sorting is done with filters.

\section{INSTRUMENT OVERVIEW}

The cryostat is mounted at the Cassegrain focus of the telescope by a rigid interface box, into which is built a spectral calibration unit consisting of transfer optics, integrating sphere, flat-field lamps, and an arc lamp. Three sections make up the cold optics: the foreoptics, the infrared slit viewer/guider, and the spectrograph. A photograph of SpeX mounted to the telescope is shown in Figure 4. The optical layout and internal view of the cryostat are shown in Figure 5.

In the foreoptics, the $f / 38.2$ beam entering the cryostat window first encounters a dichroic turret before coming to a focus. Visible-reflecting/infrared-transmitting dichroics feed the visible beam to an optical wave-front sensor package mounted on the side of the cryostat. The optical path following the cold telescope focus comprises a collimating mirror, fold mirrors, 


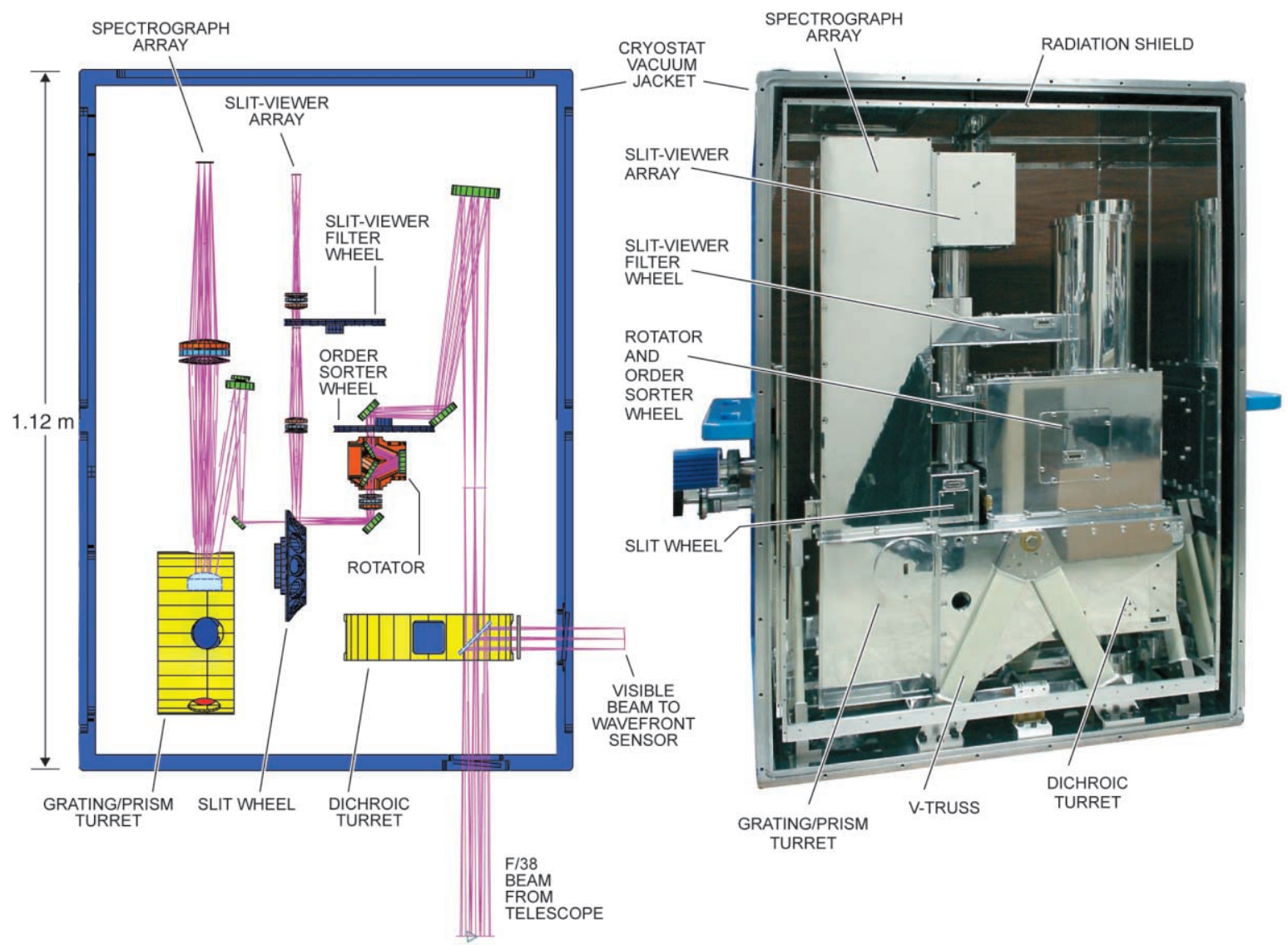

FIG. 5.-Left: Optical layout of the cryostat. Right: Photograph of the vacuum jacket and cold structure. The cryostat is shown with its front and back covers removed. Immediately inside the vacuum jacket is the closed-cycle cooled radiation shield, also shown without its front and back covers. The radiation shield is mounted to the vacuum jacket by four small fiberglass tabs. The cold structure is mounted to the vacuum jacket by four fiberglass $V$-trusses, three of which are visible in this view. The interior of the vacuum jacket, radiation shield, and cold structure are all highly polished to reduce emissivity.

order-sorter filter wheel, $12.5 \mathrm{~mm}$ cold stop, K-mirror image rotator, and camera lens. This system reimages a $60^{\prime \prime} \times 60^{\prime \prime}$ field onto a slit wheel and feeds an f/12.7 beam into the spectrograph.

The field surrounding the slit is reflected into the slit viewer by a slit mirror. The slit viewer consists of a collimating lens, $12.5 \mathrm{~mm}$ cold stop, filter wheel, camera lens, and array. A $60^{\prime \prime} \times 60^{\prime \prime}$ field is reimaged onto a $512 \times 512$ Aladdin $2 \mathrm{InSb}$ array, giving an image scale of 0.12 pixel $^{-1}$. In addition to object acquisition and scientific imaging, the slit viewer is used for infrared guiding either on spillover light from the science object in the slit or on an object in the field.

The f/12.7 beam entering the spectrograph is first folded before collimation by an off-axis parabola. The collimator forms a $24 \mathrm{~mm}$ diameter image of the telescope pupil on a grating/prism mounted in a grating turret wheel. Following dispersion, a camera lens images the spectrum onto a $1024 \times 1024$ Aladdin $3 \mathrm{InSb}$ array, which is mounted on a focusing stage. The spatial scale is $0 " 15$ pixel $^{-1}$. Because of the wide wavelength range, cross-dispersion is done with prisms, which are used in double-pass mode. The grating turret has five positions: two grating/prism cross-dispersed modes, two grating long-slit modes, and one low-resolution prism mode. All the lenses are BaF2-LiF-ZnS/Cleartran achromatic triplets, antireflection coated for $0.8-5.5 \mu \mathrm{m}$.

An IRTF-designed array controller runs both the spectrograph and imaging InSb arrays. Cryostat-mounted electronics, consisting of preamps, analog-to-digital (A/D) boards, clock, and bias boards, are fiber-optically coupled to the array controller, which resides in the telescope control room. The VME64-based array controller uses digital signal processor (DSP) boards (each containing four DSPs) and a single-board SPARC computer. One controller ("Bigdog") runs the spectrograph array, and a second ("Guidedog") runs the slit-viewer array. Table 2 summarizes the basic parameters of the instrument and arrays. A PC-based instrument computer ("Littledog") mounted on the mirror cell is used for motor and temperature control and for monitoring tasks. A graphical user 
TABLE 2

Summary of Basic InStrument Parameters

\begin{tabular}{|c|c|c|}
\hline Parameter & Spectrograph & Slit Viewer/Guider \\
\hline Detector & $1024 \times 1024$ Aladdin $3 \mathrm{InSb}$ & $512 \times 512$ Aladdin $2 \mathrm{InSb}$ \\
\hline Image scale.. & $00^{\prime \prime} 15$ pixel $^{-1}$ (along slit) & 0.12 pixel $^{-1}$ \\
\hline Field of view & $15^{\prime \prime} 0$ and $60^{\prime \prime} .0$ slit lengths & $600^{\prime \prime} 0 \times 60^{\prime \prime} 0$ \\
\hline Gain $\ldots \ldots \ldots \ldots \ldots \ldots \ldots \ldots \ldots \ldots \ldots$ & $13.0 e^{-} \mathrm{ADU}^{-1}$ & $14.7 e^{-} \mathrm{ADU}^{-1}$ \\
\hline Saturation level $^{\mathrm{a}} \ldots$ & $111,000 e^{-}$ & $88,000 e^{-}$ \\
\hline Recommended limit & $\sim 52,000 e^{-}$(5\% linear) & $\sim 37,000 e^{-}(10 \%$ linear $)$ \\
\hline \multirow[t]{2}{*}{ Read noise $\ldots . . . \ldots$} & $50 e^{-} \mathrm{rms}$ at $6 \mathrm{~Hz}$ & $65 e^{-}$rms at $3 \mathrm{~Hz}$ \\
\hline & $12 e^{-}$rms with 32 samples & $20 e^{-}$rms with 32 samples \\
\hline Dark current & $0.2 e^{-} \mathrm{s}^{-1}$ & $1.5 e^{-} \mathrm{s}^{-1}$ \\
\hline Readout rate (standard) ${ }^{\mathrm{b}}$ & $0.51 \mathrm{~s}$ per sample & $0.24 \mathrm{~s}$ per sample \\
\hline Readout rate (fast) ${ }^{\mathrm{b}} \ldots \ldots$ & $0.1 \mathrm{~s}$ per sample & $0.12 \mathrm{~s}$ per sample \\
\hline
\end{tabular}

${ }^{\text {a }}$ For a detector bias of $0.4 \mathrm{~V}$, and assuming integration time $\gg$ readout rate.

${ }^{\mathrm{b}}$ For full array. Proportionately less for subarrays.

interface (GUI) runs on a two-screen Unix workstation connected to the array controller and instrument computer by Ethernet.

\section{OPTICS}

\subsection{Concept}

The classic collimator-camera approach is adopted in the design, and the instrument comprises three major optical assemblies: foreoptics, spectrograph, and slit viewer. Each of these three assemblies contains its own collimator and camera. The main factor complicating the design is the requirement of efficient and achromatic performance across $0.8-5.5 \mu \mathrm{m}$. Allreflective designs are very expensive and beyond our budget. All-refractive designs are generally easier to fabricate and align, but they suffer from reflective losses due to the difficulty of optimizing antireflection coatings for such wide wavelength coverage. We have chosen a hybrid system of collimating mirrors and fixed camera lenses. The optics were designed using the ZEMAX optical design program from Focus Software, Inc.

\subsection{Foreoptics}

All the required focal reduction $(3.1: 1)$ is done in the foreoptics. A cold pupil image is formed in front of the narrow spectrograph slits rather than behind them. This avoids the effects of diffractive blurring of the pupil image at the cold stop by the slits and guarantees good baffling.

\subsubsection{Windows and Dichroic Beam Splitters}

The up-looking $\mathrm{CaF}_{2}$ cryostat entrance window is $76 \mathrm{~mm}$ $\times 76 \mathrm{~mm}$ to comfortably accept an $80^{\prime \prime} \times 80^{\prime \prime}$ field. This is the maximum field size that the wave-front sensor package can use. $\mathrm{CaF}_{2}$ is used because of its good transparency, low scatter, and low dispersion. A four-position turret includes two $\mathrm{CaF}_{2}$ dichroics and an open position. One dichroic cuts on at $0.8 \mu \mathrm{m}$ and the other at $0.95 \mu \mathrm{m}$; both are optimized for transmission out to $5.5 \mu \mathrm{m}$. The dichroics are placed $200 \mathrm{~mm}$ inside the entrance window. This minimizes the solid angle of warm flux viewed through the window that could be scattered at the dichroics and thus increase emissivity. Following the dichroic, the transmitted infrared beam comes to a focus at a $60^{\prime \prime} \times$ $60^{\prime \prime}(33.4 \mathrm{~mm} \times 33.4 \mathrm{~mm})$ field stop located $440 \mathrm{~mm}$ inside the entrance window. This has the advantage of defocusing dust spots on the (warm) window that can be a significant source of noise at wavelengths longer than about $3 \mu \mathrm{m}$. When the instrument is not in use, a pick-off mirror in the calibration box is positioned over the entrance window to keep it clean. The reflected optical beam comes to a focus about $80 \mathrm{~mm}$ outside a side-looking $\mathrm{CaF}_{2}$ window and on the field lens of the wave-front sensor package. A cold PK-50 blocker, mounted in the beam immediately inside this window, prevents thermal background from entering through the exit window. Both windows are tilted by $5^{\circ}$ to minimize optical ghosts.

\subsubsection{Collimator}

The beam diverging from the infrared focus is collimated by a spherical Zerodur mirror with a focal length of $473 \mathrm{~mm}$ and a diameter of $60 \mathrm{~mm}$. In order to clear the incoming beam, the collimator is tilted 4.5. Following two fold mirrors, the collimator images the telescope pupil onto a cold stop.

\subsubsection{Order-Sorter Filter Wheel and Cold Stop}

A 15-position filter wheel is located in the collimated beam immediately in front of the $12.5 \mathrm{~mm}$ diameter cold stop. This wheel contains order sorters for the spectrograph, additional filters for the slit-viewer imager, and an annular stop for spectroscopy on objects brighter than about first magnitude. Placing filters prior to the slit keeps out-of-band photons out of the spectrograph. Placing filters close to the cold stop and in the collimated beam keeps them small, maximizing the number of filters in the wheel, and eliminates the need to refocus when changing filter. The prism modes require no order sorting (or blocking), and so any filter in the slit-viewer filter wheel may 
be used for IR guiding. The cold stop is located at the front focus of the foreoptics camera lens to keep the system telecentric.

\subsubsection{Field Rotator}

A K-mirror field rotator fits into the space between the cold stop and foreoptics camera lens. The K-mirror consists of three inclined fused-silica plane mirrors mounted together in a drum. The beam enters and exits the rotator along its rotation axis through the center of two $30 \mathrm{~mm}$ diameter rotation bearings. An important goal of the rotator fabrication was to keep the optical axis and mechanical rotation axis co-aligned to within 0.1 , which is equivalent to an image wander radius of $5^{\prime \prime}$, so that an object close to the slit does not move appreciably across the $60^{\prime \prime} \times 60^{\prime \prime}$ field when the position angle is changed. (The IRTF is an equatorial telescope, so there is no image rotation during tracking.) A 3" image wander radius was achieved by building to tolerance and without any adjustment.

\subsubsection{Camera Lens}

Immediately following the rotator is the foreoptics camera lens, which reimages the telescope focal plane on the slit. The camera lens consists of a $\mathrm{BaF}_{2}-\mathrm{LiF}-\mathrm{ZnS} / \mathrm{Cleartran}$ achromatic triplet lens. Imaging is achromatic across the range $0.8-5.5 \mu \mathrm{m}$. The calculated average Strehl ratio across the field is 0.85 , 0.95 , and 0.99 at $0.9,1.65$, and $4.8 \mu \mathrm{m}$, respectively. The lenses were optimized with all spherical surfaces. To keep the mounting (and therefore the alignment) simple, optimization was not allowed to migrate the lenses more than a few millimeters apart. All three elements are $35 \mathrm{~mm}$ in diameter. The resulting lens is mounted as a vacuum-spaced triplet in a single lens mount. Scaled versions of this lens are used for the spectrograph and slit viewer. Optics for Research fabricated all the cryogenic lenses.

\subsection{Spectrograph}

The spectrograph uses a collimator and camera of equal focal lengths to reimage the slit at $1: 1$ onto the detector. The offaxis parabolic collimator places the $24 \mathrm{~mm}$ diameter pupil on the dispersing elements, and the dispersed beam is then reimaged by the triplet camera lens onto the array.

\subsubsection{Slit Wheel and Slit Mirrors}

Slit mirrors are inclined at $45^{\circ}$ to the incoming $f / 12.7$ beam to reflect a $60^{\prime \prime} \times 60^{\prime \prime}$ field into the slit viewer for IR guiding and imaging. IR guiding is done on objects in the field or on the spillover light from the science object in the slit. Consequently, the slit mirror quality must be good at the edge of the slit. Aligning the slits at $45^{\circ}$ is very advantageous for the overall instrument layout; however, it does increase the potential for scattering off the edges of the slit. To minimize scatter into the spectrograph, we chose a metal-coated substrate-type slit. A gold coating with slit aperture is lithographically applied to the front side of an antireflection-coated $\mathrm{CaF}_{2}$ substrate. Since the gold coating is less than $1 \mu \mathrm{m}$ thick, the knife edge is extremely sharp. A backside metal coating is used to absorb the 3\% reflection off the back side of the slit. At $1 \mathrm{~mm}$ thick and $25 \mathrm{~mm}$ in diameter, the substrates are flat enough for highquality focal and pupil-plane images in the slit viewer and thin enough to add little astigmatism and spherical aberration into the f/12.7 beam entering the spectrograph. Ten different slits with widths ranging from 0.3 to 3.0 and an imaging mirror are contained in the 12-position slit wheel. The slits were fabricated by Max Levy Autograph, Inc.

\subsubsection{Off-Axis Collimator Mirror}

The $f / 12.7$ beam entering the spectrograph box is collimated by a diamond-turned nickel-plated aluminum mirror made by SSG, Inc. The mirror is an off-axis parabola (OAP) with a focal length of $300 \mathrm{~mm}$ and a diameter of $44 \mathrm{~mm}$. The angle between the incident and reflected beams is $10^{\circ}$. Since the spectrograph employs slits that are matched to the telescope's diffraction limit, truncation of the beam at the slit results in diffractive expansion of the beam. For a 0.3 slit and a geometrically sized collimator, the light loss is about $12 \%$. The size of the collimator is a compromise between light loss and aberrations in the camera lens next in the light path due to the increased diameter of the optical beam. The collimator is therefore oversized by $5 \%$, which reduces the light loss to $6 \%$ without adding significantly to the aberrations.

\subsubsection{Prisms}

In general, the choice of grating (or grism) versus prism for cross-dispersion depends on the required order separation and the spectral range to be covered. Gratings usually have higher dispersion but suffer from changing efficiency when used to cover a wide spectral range. The spectral range of a grating cross-disperser is widest in first order $(m=1$; see eqs. [2] and [3]). For a grating cross-disperser used to cover the range $0.8-2.5 \mu \mathrm{m}$, the efficiency falls from a peak of about 0.7 at $1.25 \mu \mathrm{m}$ to 0.4 at $0.8 \mu \mathrm{m}$ and $2.5 \mu \mathrm{m}$ for a $5^{\circ}$ angle of incidence (Schroeder 2000). Another disadvantage of a grating is the lessthan-optimum use of detector area. When working in low orders, the order spacing significantly increases with wavelength (Schroeder 2000). For example, to cover the 0.8-2.5 $\mu \mathrm{m}$ range in six orders starting with third order at $2.2 \mu \mathrm{m}$ and using the slit length and image scale optimum for SpeX (see § 2.2) would require an array height of about 2000 pixels, twice that available, even though each order is only 100 pixels high.

Prisms have high and constant efficiency over their transparency range, but lower dispersion than gratings for the same collimated beam diameter. To compensate for the lower dispersion, prisms are used in double-pass configuration, a feature that also makes the design more compact. Order separation with wavelength is determined by the dispersion of the prism 
TABLE 3

GRATINGS

\begin{tabular}{|c|c|c|c|c|}
\hline \multirow[b]{2}{*}{ Parameter } & \multicolumn{2}{|c|}{$0.8-2.5 \mu \mathrm{m}$ Modes } & \multicolumn{2}{|c|}{ 1.9-5.5 $\mu \mathrm{m}$ Modes } \\
\hline & $\mathrm{XD}$ & Single Order & $\mathrm{XD}$ & Single Order \\
\hline Orientation & Littrow $\pm 5^{\circ}$ & Littrow $\pm 5^{\circ}$ & Littrow $\pm 5^{\circ}$ & Littrow $\pm 5^{\circ}$ \\
\hline Blaze $\lambda(\mu \mathrm{m}) \quad \ldots \ldots \ldots \ldots \ldots \ldots \ldots$ & 6.6 & 6.6 & 20.0 & 20.0 \\
\hline Working orders $\ldots \ldots \ldots \ldots \ldots \ldots \ldots$ & $3-8$ & $3-8$ & $4-10$ & $4-10$ \\
\hline Order sorter $\ldots \ldots \ldots \ldots \ldots \ldots \ldots \ldots$ & FS/ZnSe prism & Filter & $\mathrm{LiF} / \mathrm{Ge}$ prism & Filter \\
\hline Blaze angle $(\mathrm{deg}) \quad \ldots \ldots \ldots \ldots \ldots \ldots$ & 10.25 & 10.25 & 12.73 & 12.73 \\
\hline Line frequency $\left(\mathrm{mm}^{-1}\right) \ldots \ldots \ldots \ldots$ & 53.90 & 53.90 & 22.04 & 22.04 \\
\hline Working $R$ (slit width) $\ldots \ldots \ldots \ldots \ldots$ & $2000(0.3)$ & $2000(0.3)$ & $2500(0.3)$ & $2500(0.3)$ \\
\hline Beam footprint (ellipse) $(\mathrm{mm}) \ldots \ldots$ & $25 \times 30$ & $25 \times 25$ & $25 \times 42$ & $25 \times 25$ \\
\hline Grating size $(\mathrm{mm}) \ldots \ldots \ldots \ldots \ldots \ldots$ & $45.0 \times 45.0$ & $45.0 \times 45.0$ & $45.0 \times 55.0$ & $45.0 \times 55.0$ \\
\hline Grating substrate $\ldots \ldots \ldots \ldots \ldots \ldots$ & Fused silica & Fused silica & Fused silica & Fused silica \\
\hline Coating $\ldots \ldots \ldots \ldots \ldots \ldots \ldots$ & Gold & Gold & Aluminum & Aluminum \\
\hline
\end{tabular}

material rather than by geometry. In the infrared, it is very difficult to find single materials with the dispersion needed to keep the order spacing constant. Our new solution uses two prisms in series, each made from different materials. For a dispersing prism used at or near minimum deviation, the angular dispersion is given by (Schroeder 2000)

$$
\frac{d \beta}{d \lambda}=\frac{t}{a} \frac{d n}{d \lambda},
$$

where $a$ is the collimated beam diameter and $d n / d \lambda$ is the dispersion. The order spacing is made constant by matching a material in which $d n / d \lambda$ changes in such a way that order spacing increases with wavelength, with a material in which $d n / d \lambda$ changes in such a way that order spacing decreases with wavelength to exactly compensate. This results in much better use of detector area compared to grating or single prism cross dispersers.

By ray-tracing potential prism designs, we have found the best combination of materials to be fused silica (FS) and $\mathrm{ZnSe}$ in the $0.8-2.5 \mu \mathrm{m}$ range and $\mathrm{Ge}$ and $\mathrm{LiF}$ in the $1.9-5.5 \mu \mathrm{m}$ range. The average throughput of these broadband antireflection-coated dual prisms across their range of use is 0.87 and 0.80 , respectively, compared to an average of about 0.5 for a typical cross-dispersing grating.

Each dual-prism consists of two right-angled prisms mounted back to back. Because of differences in thermal contraction, the back-to-back prisms cannot be optically contacted, and a small vacuum gap of about $1.0 \mathrm{~mm}$ exists between the two prisms. To avoid interference fringes forming in the gap, the prisms are wedged by 0.3 . The prisms are circular in cross section (55 $\mathrm{mm}$ in diameter) and are mounted in thermally compensating mounts in the same way as the lenses. All the prisms were fabricated by Optics for Research.

\subsubsection{Gratings}

The gratings are illuminated in near-Littrow orientation (inplane) with a tilt of $5^{\circ}$ to allow the diffracted beam to clear the incident beam. This orientation is usually not as optically efficient as illuminating gratings in quasi-Littrow since the grating rulings are shadowed. However, the shadowing effect is not significant in SpeX because the blaze angles are small. The big advantage of near-Littrow over quasi-Littrow orientation is that the reimaged slit is not tilted with respect to detector columns, thus simplifying spectral reduction. In practice, a slight amount of tilt results from predispersion by the prisms, and some curvature is a natural consequence of the finite-length slits, but these effects are small, about 0.5 pixels over the $15^{\prime \prime}$ slit and about 2 pixels over the $60^{\prime \prime}$ slit. Custom gratings were fabricated by Zeiss and Hyperfine, Inc. The properties of the gratings are summarized in Table 3.

The system slit-limited resolving power is given by (Schroeder 2000)

$$
R=\frac{d_{1}}{r \phi D} \frac{\sin \theta_{i}+\sin \theta_{r}}{\cos \theta_{r}}
$$

where $d_{1}$ is the collimated beam diameter, $r$ is the anamorphic magnification, $\phi$ (radians) is the slit width subtended on the sky, $D$ is the telescope diameter, $\sin \theta_{i}$ is the grating angle of incidence, and $\sin \theta_{r}$ is the grating angle of reflection. For the short-wavelength gratings, $R$ is set to 2000 at the center of each order for a 0.3 wide slit and changes from about 1750 to 2250 across the array as a result of the changing angle of reflection. For the long-wavelength gratings, $R$ is set to 2500 at the center of each order for a 0.3 wide slit and changes from about 2200 to 2800 across the array as a result of the changing angle of reflection.

\subsubsection{Grating Turret}

Each of the five spectroscopy modes occupies one position in the grating turret. Spectroscopy modes are selected by rotating the continuous-drive grating turret to place a selected grating/prism combination into the collimated beam. Rotation of the turret moves spectra in the cross-dispersion direction, and fine rotation control is designed to position spectral orders 
to a precision of 1 pixel $\left(0{ }^{\prime \prime} 15\right)$, about as precise as seeing allows. In the dispersion direction, the grating turret is fixed, and positioning of spectra on the array is determined by the precision of the detented slit mechanism. The prisms are oversized so that turret rotations of $\pm 1^{\circ}$ do not vignette the beam. This flexibility permits orders in the range $0.8-5.5 \mu \mathrm{m}$ to be placed anywhere on the array to avoid bad pixels. Figure 6 shows the optical configuration of the SXD mode, consisting of back-to-back fused silica and ZnSe prisms and a grating. The LXD mode, consisting of back-to-back LiF and Ge prisms and a grating, is similar.

\subsubsection{Camera Lens}

The spectrograph camera is a scaled-up and reoptimized version of the foreoptics camera lens. Each element of the $\mathrm{BaF}_{2}-$ LiF-ZnS/Cleartran all-spherical triplet lens has a diameter of $80 \mathrm{~mm}$. Lens aberrations are small compared to diffraction.

\subsubsection{Array Focus Mechanism}

Even though the beam at the grating/prisms is collimated, small focus shifts occur when switching between different spectroscopy modes as a result of differences in flatness of the grating and prism faces. Using a flatness tolerance of $\lambda / 4$ at $0.6 \mu \mathrm{m}$ recommended by the prism vendors, a ZEMAX Monte Carlo tolerance analysis produced focus shifts of up to \pm 1 $\mathrm{mm}$ when changing grating/prism modes. The reason for the sensitivity is the number of surfaces that change when changing modes (up to nine because of the double-pass design). To compensate for these small shifts, the spectrograph array is mounted on a focus stage with a range of $\pm 2.5 \mathrm{~mm}$. In use, a focus range of about $1 \mathrm{~mm}$ is required, consistent with the tolerance analysis.

\subsection{Slit Viewer}

The slit viewer reimages the slit field at $0.8: 1$ demagnification onto the $512 \times 512 \mathrm{InSb}$ detector, using similar $\mathrm{BaF}_{2}-$ LiF-ZnS/Cleartran triplet lenses. The image scale is $0{ }^{\prime \prime} 12$ pixel $^{-1}$. All the lenses are $35 \mathrm{~mm}$ in diameter. The collimator triplet forms a $12.5 \mathrm{~mm}$ diameter pupil image immediately in front of a 15-position filter wheel. Following the filter wheel, the camera triplet focuses the field onto the detector. Imaging is achromatic across the range $0.8-5.5 \mu \mathrm{m}$. The calculated average Strehl ratio across the field is $0.85,0.95$, and 0.99 at 0.9 , 1.65 , and $4.8 \mu \mathrm{m}$, respectively.

\subsection{Calibration Box}

\subsubsection{Optics}

The calibration optics are designed to illuminate the telescope focal plane inside SpeX with a $50 \mathrm{~mm}$ diameter flat field. This is the size required to cover the longest slits $\left(60^{\prime \prime}\right)$. A $10.0 \mathrm{~mm}$ diameter exit port (field stop) of an integrating sphere serves as the uniform (Lambertian) flat field. This field is magnified

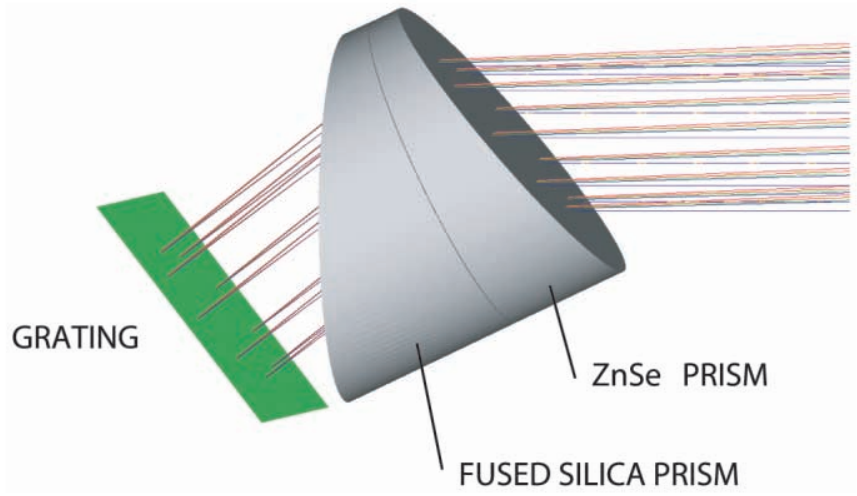

FIG. 6.-Optical layout of the SXD mode components in the grating/prism turret.

to $50.0 \mathrm{~mm}$ diameter by a $151.5 \mathrm{~mm}$ focal length $\mathrm{LiF} / \mathrm{BaF}_{2}$ achromatic doublet lens placed just under one lens focal length $(121.2 \mathrm{~mm})$ from the exit port. A concave $648 \mathrm{~mm}$ focal length spherical mirror used slightly off-axis reimages this field at $1: 1$ onto the $f / 38.2$ telescope focal plane inside the cryostat. At the same time, the mirror images the $15.8 \mathrm{~mm}$ aperture stop of the lens onto the telescope secondary mirror to create an exit pupil identical to that of the telescope (f/38.2) so that the calibration light sources illuminate the instrument identically to the telescope. There are three folding flats, one of which is mounted on a linear translation stage that moves into the telescope beam and picks off the beam from the calibration lamps when required.

\subsubsection{Integrating Sphere and Input Sources}

The $50 \mathrm{~mm}$ diameter custom-made integrating sphere from Labsphere has three entrance ports, one for a $10 \mathrm{~W}$ quartztungsten-halogen lamp $(3200 \mathrm{~K})$ and $0.1 \mathrm{~W}$ bulb (placed side by side) for the $0.8-2.5 \mu \mathrm{m}$ region, one for an infrared source $(1100 \mathrm{~K})$ for the $1.9-5.5 \mu \mathrm{m}$ region, and one for a low-pressure argon discharge lamp. With the exception of the pick-off mirror, the calibration box contains no moving parts. Argon lines from the discharge lamp are visible out to about $4 \mu \mathrm{m}$. Beyond $4 \mu \mathrm{m}$, the thermal background from the warm calibration box overwhelms the signal and wavelength calibration uses atmospheric features. Integration times of no longer than about $3 \mathrm{~s}$ are sufficient to obtain a signal-to-noise ratio $(\mathrm{S} / \mathrm{N})$ greater than 200 in the flat fields in all modes.

\subsection{Instrument Throughput}

Broadband antireflection (BBAR) multiple-layer coats for the lenses and prisms were designed and applied by Thin Film Labs. The lenses need to work efficiently across the entire $0.8-5.5 \mu \mathrm{m}$ range of the instrument. The prisms are optimized for smaller wavelength ranges, so their BBAR performance is better. All the mirrors, with the exception of the OAP, use a 
TABLE 4

Estimated Throughput of 0.8-2.4 $\mu \mathrm{m}$ Cross-Dispersed (SXD) Mode at $1.7 \mu \mathrm{m}$

\begin{tabular}{|c|c|c|}
\hline Element & Efficiency & Explanation \\
\hline Primary mirror $\ldots \ldots \ldots \ldots$ & 0.99 & Aluminum on Cervit \\
\hline Secondary mirror $\ldots . . . .$. & 0.99 & Aluminum on $\mathrm{SiC}$ \\
\hline Window $\ldots \ldots \ldots \ldots \ldots . . . . . .$. & $0.97^{2}$ & $0.4-5.5 \mu \mathrm{m}$ single-layer protection coat $\mathrm{CaF}_{2}$ \\
\hline Dichroic $\ldots \ldots \ldots \ldots \ldots . . . . .$. & $(0.9)$ & $\mathrm{CaF}_{2}$ substrate \\
\hline Mirror collimator ......... & 0.99 & Protected silver on Zerodur \\
\hline Fold mirrors $(2) \ldots \ldots \ldots$ & $0.98^{2}$ & Protected silver on silica at $45^{\circ}$ \\
\hline Cold stop $\ldots \ldots \ldots \ldots \ldots$ & 0.95 & Mask edge of $3.0 \mathrm{~m}$ primary mirror \\
\hline $\mathrm{K}$-mirror (3) rotator ...... & $0.98^{3}$ & Protected silver on silica at $45^{\circ}$ \\
\hline \multirow[t]{3}{*}{ Triplet lens $\ldots \ldots \ldots \ldots \ldots$} & $0.975^{2}$ & 0.8-5.5 $\mu \mathrm{m}$ BBAR on $\mathrm{BaF}_{2}$ (witness sample) \\
\hline & $0.975^{2}$ & 0.8-5.5 $\mu \mathrm{m}$ BBAR on $\mathrm{LiF}$ \\
\hline & $0.95^{2}$ & 0.8-5.5 $\mu \mathrm{m}$ BBAR on $\mathrm{ZnS} /$ Cleartran (witness sample) \\
\hline Fold mirror (1) & 0.98 & Protected silver on silica at $45^{\circ}$ \\
\hline Slit ............. & $0.97^{2}$ & $0.8-5.5 \mu \mathrm{m} \mathrm{BBAR}$ on $\mathrm{CaF}_{2}$ substrate at $45^{\circ}$ \\
\hline Fold mirror (1) & 0.98 & Protected silver on silica at $45^{\circ}$ \\
\hline Mirror collimator ......... & 0.99 & Diamond-turned Al, gold coat \\
\hline \multirow{4}{*}{ Double-pass prism ........... } & $0.975^{2}$ & $0.8-2.5 \mu \mathrm{m}$ BBAR on $\mathrm{ZnSe}$ at $40^{\circ}$ \\
\hline & $0.98^{2}$ & $0.8-2.5 \mu \mathrm{m}$ BBAR on $\mathrm{ZnSe}$ at $0^{\circ}$ (witness sample) \\
\hline & $0.99^{2}$ & $0.8-2.5 \mu \mathrm{m}$ BBAR on fused silica at $0^{\circ}$ (witness sample) \\
\hline & $0.985^{2}$ & $0.8-2.5 \mu \mathrm{m}$ BBAR on fused silica at $40^{\circ}$ \\
\hline Grating $\ldots \ldots \ldots \ldots \ldots \ldots$ & 0.75 & Estimate at peak, near-Littrow $5^{\circ}$ tilt \\
\hline \multirow[t]{3}{*}{ Triplet lens $\ldots \ldots \ldots \ldots \ldots$} & $0.975^{2}$ & 0.8-5.5 $\mu \mathrm{m}$ BBAR on $\mathrm{BaF}_{2}$ (witness sample) \\
\hline & $0.975^{2}$ & 0.8-5.5 $\mu \mathrm{m}$ BBAR on $\mathrm{LiF}$ \\
\hline & $0.95^{2}$ & 0.8-5.5 $\mu \mathrm{m}$ BBAR on $\mathrm{ZnS} /$ Cleartran (witness sample) \\
\hline Detector ......... & 0.95 & Typical of best Aladdin arrays at $1.7 \mu \mathrm{m}$ \\
\hline Total throughput & 0.29 & $0.8-2.5 \mu \mathrm{m}$ cross-dispersed mode at $1.7 \mu \mathrm{m}$ \\
\hline
\end{tabular}

protected-silver reflection coating (FSS-99) by Denton Vacuum. The OAP from SSG, Inc., uses the company's standard vacuum-deposited bare gold coating. The slit mirrors also use gold coatings.

The throughput in the prism, SXD and LXD modes, was measured on an $\mathrm{A} 0 \mathrm{~V}$ star through the 3".0 wide slit to minimize seeing losses. Figure 7 is a plot of the throughput in the prism and SXD modes. In the telluric bands, the effects of atmospheric transmission have been removed by using a model of the atmosphere. The estimated throughput of SpeX at $1.7 \mu \mathrm{m}$ in the 0.8-2.4 $\mu \mathrm{m} \mathrm{XD}$ mode is given in Table 4. The peaks of the SXD blaze function were designed to be at $6.6 \mu \mathrm{m} \mathrm{m}^{-1}$ (see eq. [1]); however, the two custom gratings made for this mode have measured first-order blaze peaks at about $6.15 \mu \mathrm{m}$. Consequently, the blaze peak in higher orders is shifted to slightly shorter wavelengths than planned. Allowing for this shift, the measured throughput $(0.30$ at $1.52 \mu \mathrm{m})$ and estimated throughput $(0.29$ at $1.7 \mu \mathrm{m})$ are in good agreement. The decrease in throughput at shorter wavelengths is probably due to the combination of decreasing quantum efficiency $(\mathrm{QE})$ of the array (including its own single-layer antireflection coating; see $\S 8.1$ ) and decreasing efficiency of the BBAR coatings on the transmissive optics (although witness samples indicate good performance of the lens and prism BBAR coatings down to $0.8 \mu \mathrm{m})$. The average throughput of the SXD mode in the range $0.8-2.4 \mu \mathrm{m}$ is about 0.18 . By contrast, the average throughput of the prism mode is about double the SXD mode with a peak

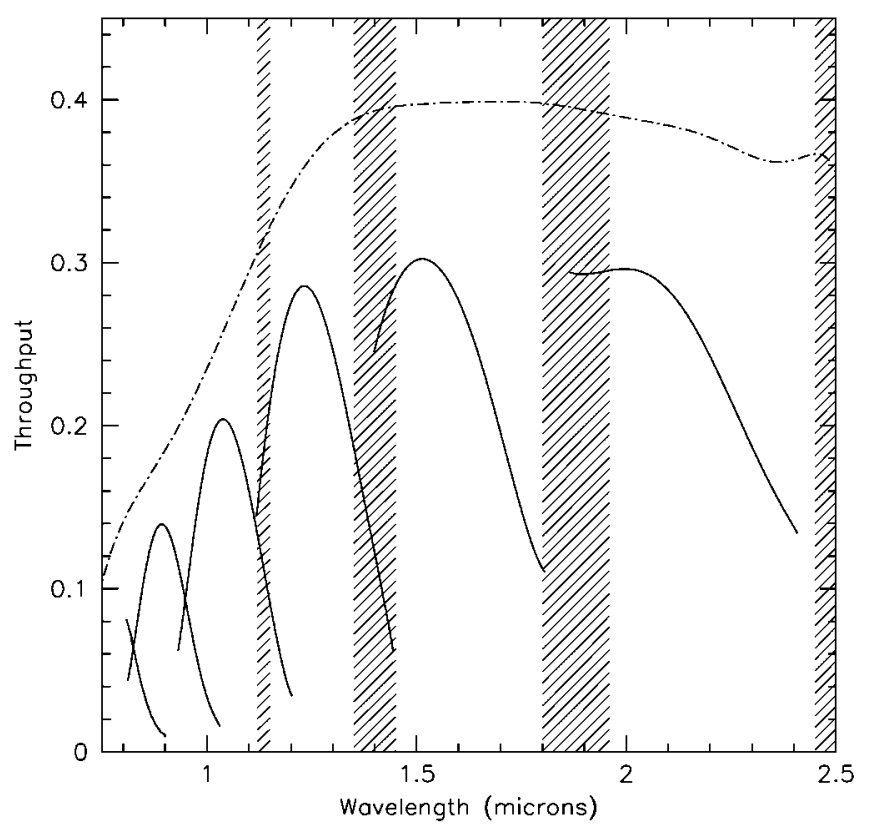

FIG. 7.-Throughput of the spectrograph, measured in the prism (dash-dotted line) and SXD (black line) modes. 
of 0.4 (at $1.7 \mu \mathrm{m}$, where the array antireflection coating is optimized), since it does not require a grating for dispersion. Because of the opacity of the atmosphere in the $2.4-5.5 \mu \mathrm{m}$ region, it is not possible to produce a continuous throughput plot for the LXD mode equivalent to Figure 7. By measuring the throughput in clear parts of the atmosphere, we find an average throughput in the LXD mode of 0.15 with order peaks at 0.2. This is slightly lower than the SXD mode and is consistent with decreasing QE toward longer wavelengths (see $\S 8.1$ ).

Assuming nominal performance of the optics and the array, the average throughput of the slit viewer should be about 0.25 . This is lower than for an optimized camera because of the additional losses in the foreoptics. The foreoptics alone have an estimated throughput of 0.66 . We measure an average throughput of 0.20 at $J, H$, and $K$, slightly lower than expected. This is probably due to a slightly lower than normal QE of the engineering-grade array used (see $\S 8.1$ ).

\subsection{Stray Light and Baffling}

Stray light and thermal background from the sky and telescope are controlled by using a cold Lyot stop in the foreoptics in front of the spectrograph and slit viewer. The spectrograph and slit viewer each contain their own cold stops.

A number of further measures are taken to control stray light and thermal background from inside the cryostat. (An unfiltered $5.5 \mu \mathrm{m}$ cutoff detector detects $\sim 10^{4}$ times as many thermal photons from an object at room temperature compared to an unfiltered $2.5 \mu \mathrm{m}$ cutoff detector.) Except at the entrance window and wave-front sensor exit window, the light paths are entirely enclosed in aluminum enclosures consisting mostly of baffled tubes. These enclosures are designed to prevent stray light and thermal background from cryostat components warmer than about $80 \mathrm{~K}$ (such as the warm vacuum jacket, drive shafts, and wires) from entering the light path, where it can be scattered toward the detectors. A goal of the design was to keep the instrument background below the detector dark current $\left(\sim 0.2 e^{-} \mathrm{s}^{-1}\right)$ because in several observing modes, the noise is limited by dark current. Since the light path needs to be easily assembled and disassembled, and efficiently evacuated, the structures were built using re-entrant tubes containing labyrinth-like evacuation ports. Drive shafts enter through rotary light shields, and all access covers have tongue-and-groove seals.

Because of the wide wavelength coverage of SpeX, large changes in either object or sky brightness can occur across spectra. For example, the change in sky brightness across the LXD modes is $\sim 10^{4}$. Consequently, any ghost reflection off a bright part of the spectrum onto a fainter region can seriously degrade data quality. The two main areas of concern are narcissus ghosts from the camera lens and prisms. Ray-tracing of the former shows that these ghosts are grossly out of focus and do not pose a problem. The latter are avoided by tilting the spectrograph array by 1.0 , which is sufficient to redirect any ghost reflections off the prisms but small enough to incur no detectable defocus across the array. The measured ghosting in SpeX is at the level of a few percent and is normally removed when the sky beam is subtracted.

\section{CRYOSTAT}

\subsection{Mechanical Design}

The vacuum jacket consists of a four-sided center section weighing about $90 \mathrm{~kg}$ onto which two large O-ring sealed covers, each weighing about $35 \mathrm{~kg}$, are bolted. The four-sided center section is electron-beam welded from four plates of 2219-aluminum (see Fig. 5). The external dimensions of the vacuum jacket are $112 \mathrm{~cm} \times 79 \mathrm{~cm} \times 61 \mathrm{~cm}$. Since no hard connections are made with the vacuum jacket except at the rigid $25 \mathrm{~mm}$ thick top plate, stability concerns are limited to keeping the flexure well below the yield strength of the aluminum and to keeping the flexure of shafts connecting to warm motors mounted on the vacuum jacket within the specifications of the flexible couplings. This is easily achieved using a vacuum jacket wall thickness of $12.5 \mathrm{~mm}$. A spreader bar has been added between the two large covers, without which the wall thickness would have to be considerably larger. The maximum observed deflection upon evacuation is $2 \mathrm{~mm}$.

SpeX is attached to the telescope multiple instrument mount by a rigid interface box mounted to the top plate of the vacuum jacket (see Fig. 4). A spectral calibration unit is built into this box. The walls of the calibration box are $25 \mathrm{~mm}$ thick, and the box is $28 \mathrm{~cm}$ deep. The top plate of the vacuum jacket contains the entrance window, liquid nitrogen fill port, and mount for the closed-cycle cooler. The cryostat-mounted electronics box and wave-front sensor box are mounted on opposite sides of the cryostat center section.

The 6061-T6-aluminum cold structure is supported on four V-shaped fiberglass support trusses that connect to the cold structure near the center of gravity (see Fig. 5). The base of the support trusses is bolted directly to the inside surface of the rigid top plate of the center section. In the interests of stiffness, the cold structure consists of a series of boxes bolted to a backbone that provides cooling and support. The total weight of the cold structure is $140 \mathrm{~kg}$. The cold structure is enclosed by a $3 \mathrm{~mm}$ thick aluminum radiation shield. The $30 \mathrm{~kg}$ radiation shield is supported separately by four fiberglass tabs. The total weight of the instrument at Cassegrain focus is $478 \mathrm{~kg}$ (not including the wave-front sensor).

\subsection{Mechanisms}

There are seven cryogenic mechanisms in the cryostat. We use external warm motors with vacuum feed-throughs in preference to internal cold motors because it is cheaper and, in our view, involves less risk. The advantage of being able to position mechanisms manually for testing, and in case of failure, is highly valued. The motors are mounted on one of the two covers, and the optics and detectors are accessed by removing 
the opposite cover. Motors need not be disassembled for most troubleshooting inside the cryostat.

The mechanisms fall into three different categories: discrete position wheels with detents, continuously variable wheels, and a linear flex stage. Since failure rates for mechanical limit switches in previous IRTF instruments have been unacceptably high, we decided to use noncontact Hall effect devices for position sensing. Each of the wheels is equipped with one stationary Hall effect sensor. Wheel positions are encoded by placing magnets on the edge of the wheels. Opposite polarities mark home positions and discrete (element) positions. The outputs of the Hall effect sensors are passed through comparator circuits and function much like limit switches.

The dichroic turret, order-sorter filter wheel, slit wheel, and slit-viewer filter wheel are all wheels with detented positions that are driven by compliant worm-gear mechanisms that use 400 step revolution ${ }^{-1}$ stepper motors. In order for the detent to properly locate the wheel, some angular compliance is provided by allowing the worm to slide along the supporting shaft, thereby providing the angular "slop" for detent engagement. The detent rolls on the periphery of the wheel and is forced into the detent with a spring. The array focus stage employs a continuous antibacklash linear position mechanism with an aluminum alloy flex stage providing $\pm 2.5 \mathrm{~mm}$ of focus travel. The flex stage itself is fabricated using electric discharge machining and provides compliance in one direction and very high stiffness in all others through the use of thin sections. The stage is driven with a screw that in turn is coupled with a worm and worm gear to a motor shaft connected to a 400 step revolution $^{-1}$ stepper motor. The grating turret and K-mirror image rotator mechanisms require continuous rotary motion and employ worm and antibacklash worm-gear mechanisms driven by Animatics DC servos with 4000 steps revolution ${ }^{-1}$ to achieve high-resolution positioning. In both mechanisms, a position is determined by counting steps from a home position indicated by a Hall effect sensor.

\subsection{Cryogenic Design and Performance}

The cooling scheme adopted in SpeX is a simple and conservative design that meets all the temperature and cooling requirements. Cooling the cold structure to liquid nitrogen $\left(\mathrm{LN}_{2}\right)$ temperatures is adequate to keep the instrument background below the best expected InSb detector dark current of $0.1 e^{-} \mathrm{s}^{-1}$, since all but a half-viewing angle of $12^{\circ}$ can be shielded with a baffle at the temperature of the detector $(30 \mathrm{~K})$. A closed-cycle cooler is used to cool a radiation shield surrounding the cold structure, and the cold structure is attached to an $\mathrm{LN}_{2}$ can that provides a very stable temperature. The use of an $\mathrm{LN}_{2}$ can also speeds cooling. The surface areas of the vacuum jacket, actively cooled radiation shield, and cold structure are $4.0,3.5$, and $2.0 \mathrm{~m}^{2}$, respectively. The actively cooled shield need only be cooled to a temperature that reduces the radiation load on the cold structure down to the level of the other parasitic loads, such as the G10 fiberglass support trusses, which can be accommodated by the $\mathrm{LN}_{2}$ can.

We use a two-stage 1050-CTI cooler to cool the radiation shield and detectors. The first stage has a rated cooling of about $70 \mathrm{~W}$ at $80 \mathrm{~K}$, and the second stage has a rated cooling of about $8 \mathrm{~W}$ at $20 \mathrm{~K}$. The first stage cools a highly polished $3 \mathrm{~mm}$ thick 6061-aluminum cold shield to less than $100 \mathrm{~K}$. The resulting radiation load from the radiation shield onto the cold structure is less than $0.5 \mathrm{~W}$. The estimated heat load on the cold structure consists of $3 \mathrm{~W}$ from the G10 support trusses, $1 \mathrm{~W}$ from the motor shafts, $1 \mathrm{~W}$ from the wiring, $2 \mathrm{~W}$ from the entrance window, and $2 \mathrm{~W}$ from penetrations in the radiation shield. Since the heat load from the radiation shield is so small compared to the other parasitic heat loads, any normal variation in the closed-cycle cooling to the radiation shield has little effect on the temperature of the enclosed cold structure. Larger temperature excursions of typically $0.5 \mathrm{~K}$ result from changes in the level of $\mathrm{LN}_{2}$ as a result of boil-off, but this is too small to have any measurable impact on instrument performance (e.g., focus or instrument background). The calculated hold time of the 10 liter capacity $\mathrm{LN}_{2}$ can compares well with the measured hold time of about $48 \mathrm{hr}$. The bulk of the $90 \mathrm{~kg}$ cold structure cools to $80 \mathrm{~K}$ in about $24 \mathrm{hr}$, at which time all the mechanisms function normally. Since there is little conduction cooling through the bearings of the filter wheels and turrets, the surfaces of these wheels are painted black to speed cooling by radiation. The instrument background measured at the spectrograph detector falls to $300 e^{-} \mathrm{s}^{-1}$ at $24 \mathrm{hr}$, $5 e^{-} \mathrm{s}^{-1}$ at $48 \mathrm{hr}$, and bottoms out at $\sim 0.1 e^{-} \mathrm{s}^{-1}$ after $72 \mathrm{hr}$. About 150 liters of $\mathrm{LN}_{2}$ is required to reach the operational temperature at $48 \mathrm{hr}$.

The spectrograph and slit-viewer arrays are located close together, at the end of the cryostat opposite to the closed-cycle cooler. The array mounts are cooled via a $0.8 \mathrm{~m}$ long copper strap with a cross-sectional area of $25 \mathrm{~mm}^{2}$. Each of the array mounts, including cold baffle snouts, weighs about $0.9 \mathrm{~kg}$ and takes $11 \mathrm{hr}$ to cool to the operating temperature of $30 \mathrm{~K}$. Using heater resistors, each mount is controlled to $30.00 \pm 0.01 \mathrm{~K}$ by a Lakeshore model 330 temperature controller. Warmup also takes $48 \mathrm{hr}$. After turning off the closed-cycle cooler and blowing out the $\mathrm{LN}_{2}$, warm-up of the cryostat is accelerated by circulating warmed dry air through the $\mathrm{LN}_{2}$ can.

\section{ARRAY CONTROLLER AND DATA SYSTEM}

The array controller for SpeX can be separated into six major hardware components: a VME64 instrument controller, a cryostat-mounted electronics box (containing preamps, A/D converters, clock, and bias boards), an array electronics power supply, an embedded computer/motor controller, a temperature controller, and a workstation displaying the SpeX GUI. The VME64 instrument controller consists of two independent computers. One computer, known as Bigdog, runs the spectrograph $1024 \times 1024 \mathrm{InSb}$ array, and a second, known as Guidedog, 
runs the IR slit viewer/guider $512 \times 512 \mathrm{InSb}$ array. For motor and temperature control, and for monitoring tasks, Bigdog and Guidedog communicate with the embedded computer known as Littledog. The location and interconnections of these components are illustrated in Figure 8.

\subsection{VME64 Instrument Control Computer}

Bigdog and Guidedog are housed in a VME64 rack mount located in the telescope control room. Other than the shared chassis and power supply, the two controllers are not coupled. The chassis has two independent VME backplanes, and each controller operates independently. Each controller houses a workstation-class single-board computer running the Unix/ Solaris 2.6 operating system, DSP-based single-board computers, a fiber-optic link to the cryostat-mounted electronics, and a board for time-stamping data. The IXZ4444 DSPs perform the time-critical tasks of pixel clocking, pixel data acquisition, and low-level pixel processing. The Unix computer performs upper level control functions, including loading code into the DSPs and commanding them, controlling the instrument via the GUI, and higher level data processing and display.

Guidedog is identical to Bigdog except that its smaller array requires only one IXZ4444 DSP board and one fiber board.

Each IXZ4444 DSP board contains two independent groups of two DSPs. The first group of "clock" DSPs outputs clocking and bias patterns in the form of 32 bit words to the fiber channel interface board, which then transmits these patterns across the optical fiber to the clock and bias boards located at the cryostat. The second group of "buffer" DSPs receives 16 bit digitized pixel values from the A/D converters at the cryostat via the optical fiber and fiber channel interface board. The buffer DSPs perform the pixel processing steps of co-adding and time stamping.

In Bigdog, two DSP boards are used to increase the pixel processing speed. One board clocks and buffers the top half (quadrants 1 and 2) of the $1024 \times 1024$ array while the second clocks and buffers the bottom half (quadrants 3 and 4). This architecture allows the full array to be read out in a minimum integration time of $0.1 \mathrm{~s}$ and proportionately shorter for subarrays.

The architecture also allows data to be streamed onto the local disk without interruption and with minimal gaps between images (or spectra). This is called "movie mode." In this mode, images are accumulated in one of the DSP board's SRAM modules. When this location is full, storage is switched to the second SRAM and the first SRAM is read out. By alternating between the two SRAMs, images can be streamed onto the VME bus for storage onto the local hard disk. Each SRAM readout is saved as a three-dimensional FITS image. The maximum storage rate for a $1024 \times 1024$ image is $2 \mathrm{~Hz}$. Movie mode is normally used in the prism configuration where a 0.8-2.5 $\mu \mathrm{m}$ spectrum is matched to quadrant two of the spectrograph array so that small subarrays can still acquire a large spectral range. In this mode a $512 \times 100$ subarray can be streamed to the local hard disk at about $10 \mathrm{~Hz}$ (and proportionately faster for smaller subarrays) for occultation spectroscopy. The only limit to the occultation time is then the storage capacity of the disk.

Guidedog works in a similar fashion except that the one quadrant $512 \times 512$ array requires only one DSP board. Images from Guidedog can be streamed to disk independently of Bigdog. For occultations, this allows spillover at the slit to be monitored.

The array data are read out over the VME bus, formatted into FITS, displayed on the X-Windows graphics display, and stored on disk. For the normal mode of observing, where integration times are longer than a few seconds and the overhead in storing data is relatively short, data are sent across the Ethernet LAN for storage on the larger capacity network disks (80 Gbytes), rather than stored in the local disks on Bigdog and Guidedog (each 8 Gbytes).

\subsection{Cryostat-mounted Electronics}

The cryostat-mounted electronics provides for the complete control of array readout and digital conversion of pixel analog signal values. The spectrograph and guider electronics are housed in a single 19 inch rack-mount chassis that is mounted to the side of the cryostat to keep the high-frequency analog lines to the arrays as short as possible. Array clocking patterns and programmable biases (most of the biases are hard-wired) are transmitted over the fiber-optic link from the clock DSPs and passed down a backplane into a clock-level-shifting circuit that translates the TTL signals into 12 bit digital-to-analog voltages to drive the arrays. Each of the 32 spectrograph array outputs and each of the eight guider array outputs are conditioned by a preamplifier circuit and output to its own $2 \mathrm{MHz}$, 16 bit A/D converter. The converted digital values are then transferred through another backplane onto the fiber-optic interface board for transmission to the pixel-buffering DSPs.

\subsection{Embedded Computer and Motor Controller}

Littledog is an embedded computer containing peripheral electronic boards for motor control, temperature monitoring, spectrograph calibration box control, AC power control, and monitoring. It operates under command from Bigdog and Guidedog using the telescope's Ethernet LAN. To keep the analog lines short, Littledog is located on the mirror cell next to the cryostat. Littledog is an i486/100 MHz PIC/ISA computer with 24 Mbytes RAM, a 10 Mbyte s $^{-1}$ Ethernet, and an IDE 500 Mbyte hard disk. The operating system is Linux. The chassis also houses power supplies for stepper and servo motors, as well as the stepper motor driver modules.

\subsection{Observing Workstation}

Observers at the telescope log in to Bigdog and Guidedog from the facility workstation located in the observer's area. 


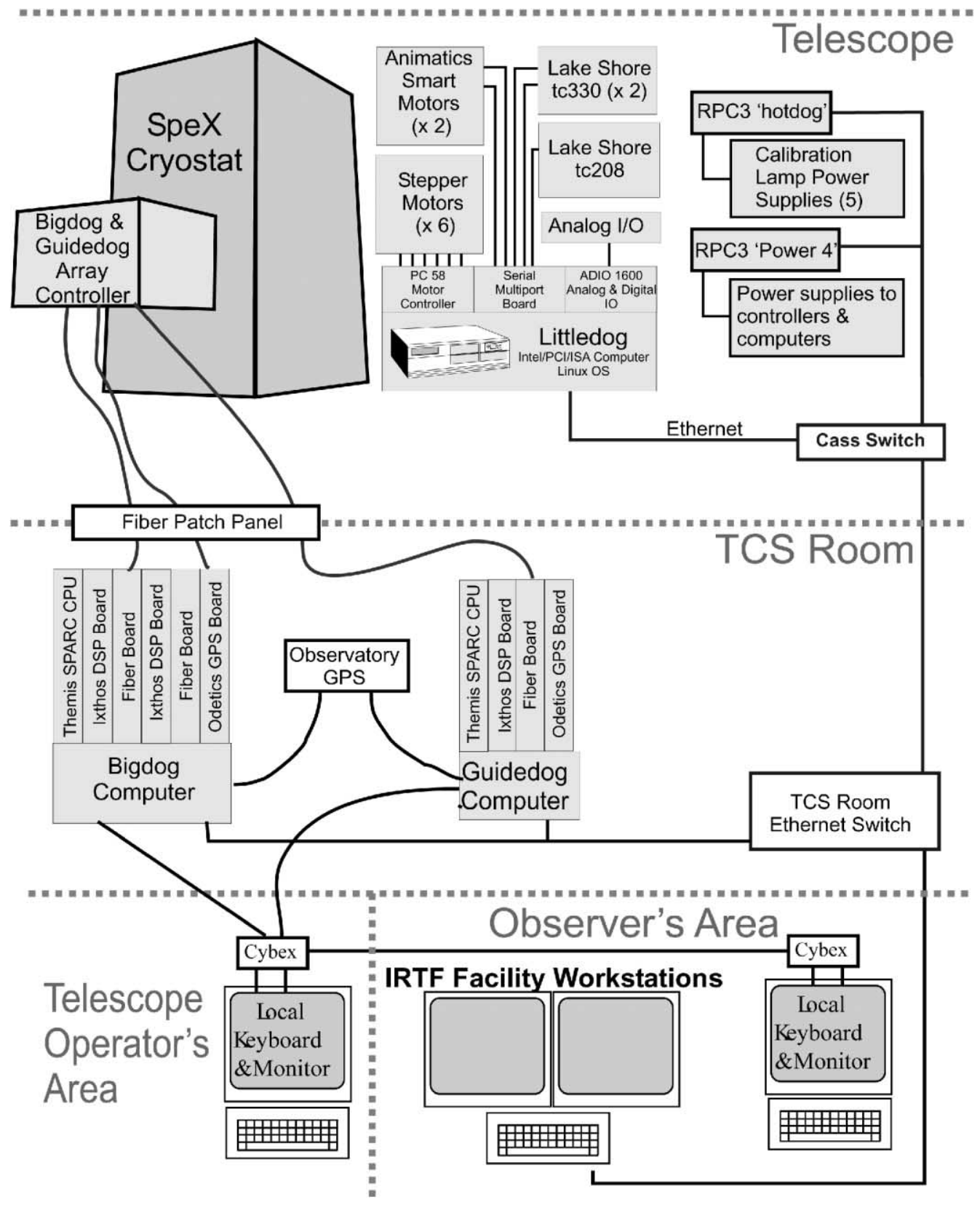

FIG. 8.-Locations and interconnections of the cryostat, computers, and array controller components.

The SpeX GUIs run on Bigdog and Guidedog, but the display windows are exported to the observer's workstation. This workstation has two screens, one for Bigdog and one for Guidedog. By logging into Bigdog and Guidedog, remote observing can be done from any appropriate workstation connected to the Ethernet interface.

\section{SOFTWARE}

\subsection{Instrument Control}

The instrument control software consists of four major components: the instrument control (IC) application, which runs on
Bigdog and Guidedog; the motor control and monitoring application, which run on Littledog; the X-Windows user interface (XUI); and the quick-look Data Viewer.

\subsubsection{Instrument Control (IC)}

Independent versions of the instrument-control application run on the Bigdog and Guidedog computers. Its purpose is to run the command parser, to run the data acquisition task, and to monitor Littledog functions. The command parser accepts user commands from the GUI (XUI) and executes them. Some commands are routed to the data acquisition task and some to 
Littledog. The acquisition task is responsible for controlling the DSP clocking and data buffering. When the data are ready, the acquisition tasks assembles them into FITS images, saves them on disk, and/or sends the them to Data Viewer for display. A monitoring task makes the Littledog status (motors and temperatures) available at the IC for display by the XUI. The IC application is written in $\mathrm{C}$.

\subsubsection{Motor Control and Monitoring}

A suite of applications runs on Littledog to initialize, position, and monitor the eight motors and position sensors and to monitor the temperature controllers and remote power control units. All the code is written in C.

\subsubsection{X-Windows User Interface}

The X-Windows user interface (XUI) provides an easy-touse GUI to control the IC using various icons, buttons, menus, and software widgets. It also provides a simple way to set up macro files built from the IC's command set. For the benefit of IRTF observers, the look and feel of the SpeX GUI was designed to be very similar to those of the other IRTF facility instruments. Like the IC, there are two independent XUIs, one for Bigdog and one for Guidedog. The ICs allow multiple versions of the XUI to execute, which allows for remote support of observers by IRTF staff or remote participation by observers. SpeX has been operated successfully from the US mainland and Europe. The XUI is written in C and uses the GTK+ widget library. Sample screens from Bigdogxui and Guidedogxui can be viewed at the SpeX Web page. ${ }^{4}$

\subsubsection{Data Viewer}

Data Viewer (DV) is a general-purpose FITS viewer for viewing images and spectra. In addition, it incorporates functions allowing interaction with the IC/XUI for guiding, telescope pointing, and setting subarrays. In normal operation, DV is displayed side by side with Bigdogxui and Guidedogxui. DV can also be used as a stand-alone FITS viewer. DV is written in $\mathrm{C}$ and uses the GTK+ widget library.

\subsection{Observing Modes}

SpeX has three fundamental observing modes, each of which can be selected with the XUI: basic, slow guide, and movie mode.

\subsubsection{Basic}

Basic is the fundamental array data acquisition mode. A "GO" command in Bigdogxui takes a spectrum, and a GO in Guidedogxui takes an image, each with the following adjustable parameters: on-chip integration time, co-additions (co-adds), nondestructive reads, array clock speed, telescope nod pattern (beam A, B, or beam A followed by beam B), and number of

\footnotetext{
${ }^{4}$ http://irtfweb.ifa.hawaii.edu/Facility/spex.
}

cycles (repeats of a GO command). There is no intrinsic limit to the maximum on-chip integration time, but it is set in software to $1800 \mathrm{~s}$. The minimum integration time is $0.1 \mathrm{~s}$, which is proportionately less for subarrays.

\subsubsection{Slow Guide}

Slow guide is the guiding mode of the infrared slit viewer in Guidedogxui. It takes images continuously and sends them to the DV and to a stand-alone video display for fast display. The slow guide mode includes several adjustable parameters: integration time, guide algorithm (peak or centroid), gain (fraction of calculated telescope offset to be moved), guiding on or off, sky subtraction on or off, and guiding in both beams or just A or B-all of these can be changed on the fly to fine tune the guiding. Slow guiding sends telescope offsets across the Ethernet to the telescope control system at a maximum frequency of about $1 \mathrm{~Hz}$. This speed is adequate for telescope tracking corrections. Anything faster requires correction through an optical fiber connection to the tip-tilt hexapod secondary mirror. This "fast guide" mode is not yet implemented.

\subsubsection{Movie}

Movie mode is available in both Bigdogxui and Guidedogxui. It includes all the parameters of basic mode with the addition that the GO command takes images continuously and streams the data to disk until stopped by the user (as described in $\S 6.1$ ). Since Bigdog and Guidedog are independent, spectra and images can be acquired simultaneously. Movie mode is designed for occultations, but it can also be used to construct high spatial resolution images on bright objects by selecting and then combining images with the best seeing from movie sequences.

\subsection{Spectral Reduction}

Data are viewed at the telescope as array images on the observing workstation. The IDL GUI-based spectral reduction package Spextool can be run on another workstation. Using observer-selected files, Spextool produces one-dimensional spectra that have been flat-fielded, wavelength calibrated, and corrected for telluric absorption. For the XD modes, Spextool also merges the cross-dispersed orders. The design of Spextool and the data reduction procedure are described in a paper by M. C. Cushing et al. (2003, in preparation). A new method for correcting near-infrared spectra for telluric absorption using A0 V stars, which is incorporated into Spextool, is discussed in Vacca, Cushing, \& Rayner (2003). Spextool can be downloaded from the SpeX Web page at the IRTF Web site.

\section{LAB TESTING}

\subsection{Array Testing and Selection}

SpeX received its arrays from the NASA Planetary Astronomy Infrared Detector Array Infrastructure Project (PAIDAI), which was led by Dr. Mike Mumma of the Goddard Space 


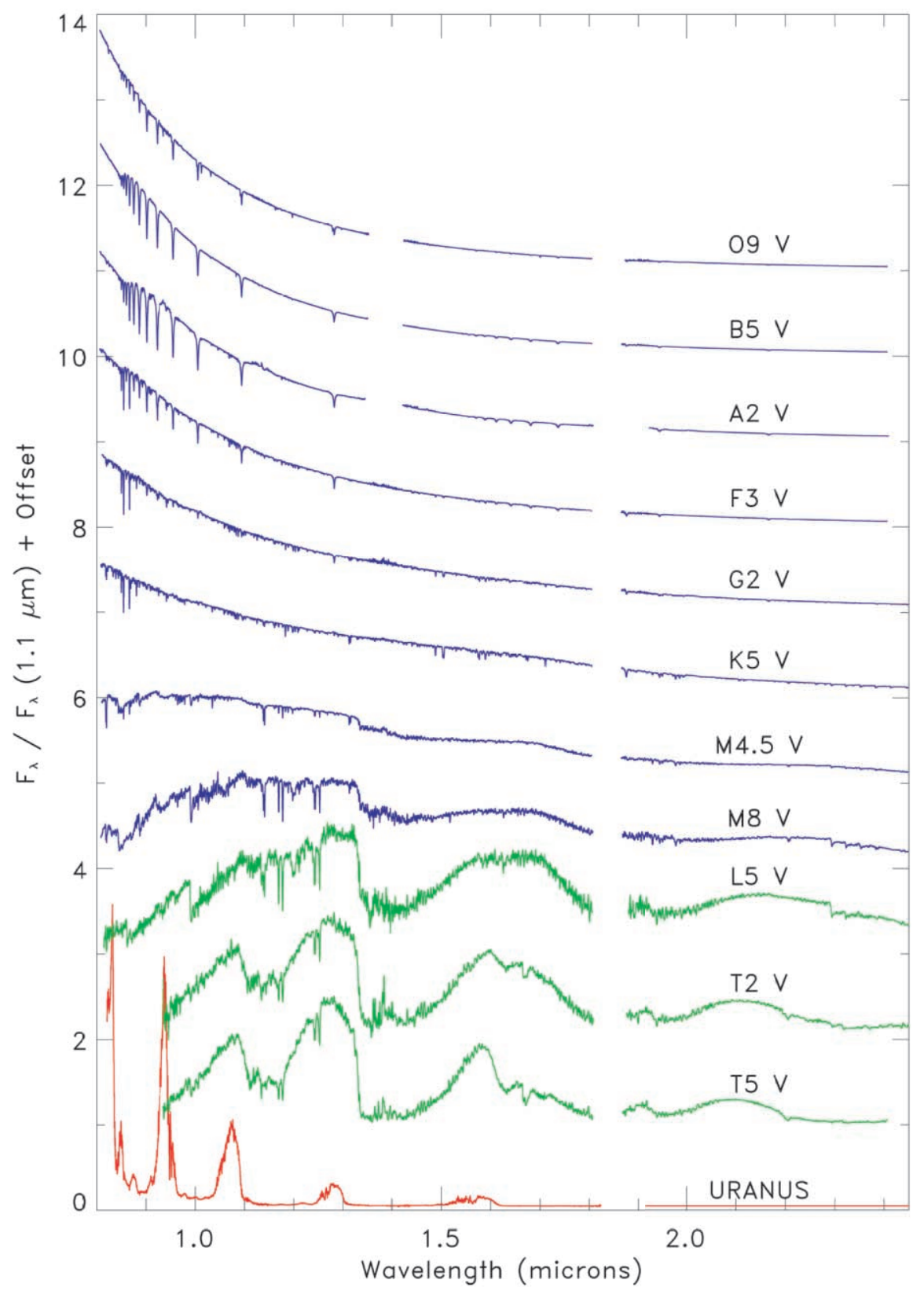

FIG. 9. - 0.8-2.4 $\mu \mathrm{m}$ stellar to substellar temperature sequence obtained with the SXD mode. Main-sequence stars are plotted in blue, brown dwarfs in green, and the planet in red.

Flight Center. PAIDAI funded a foundry run of Aladdin $1024 \times 1024$ and $512 \times 512$ arrays at Raytheon Infrared Operations (RIO). As a result of tests at RIO and PAIDAI partner NOAO, we selected the Aladdin 3 array SCA412202. Aladdin 3 devices employ an improved (SBRC-206) multiplexer and have generally better read noise, speed, and reduced odd/even column structure compared to Aladdin 2 devices (SBRC-152 multiplexer). All Aladdin arrays are coated with a single-layer $\mathrm{SiO}$ antireflection (AR) coat on InSb, optimized at $1.7 \mu \mathrm{m}$. Reflection losses at the array are at minimum a few percent at $1.7 \mu \mathrm{m}$, increasing to about 0.30 at $5.0 \mu \mathrm{m}$ and 0.36 at
$0.85 \mu \mathrm{m}$ (where the single-layer AR coat has a maximum reflectance equal to that of bare InSb). Measured QEs of the best Aladdin arrays are typically about 0.95 at $1.7 \mu \mathrm{m}$. Testing of SCA412202 in our lab test cryostat, and later in the instrument on the telescope, confirmed the expected performance at $30 \mathrm{~K}$ and $0.4 \mathrm{~V}$ bias: dark current $0.2 e^{-} \mathrm{s}^{-1}$, a read noise of $50 e^{-} \mathrm{rms}$ (reset-read-read) measured at a readout rate of $6 \mathrm{~Hz}$, which is reduced to about $12 e^{-}$at slower speeds and with Fowler multiple sampling (implemented with up to 32 nondestructive reads at the beginning of an integration followed by another 32 nondestructive reads at the end of the integra- 


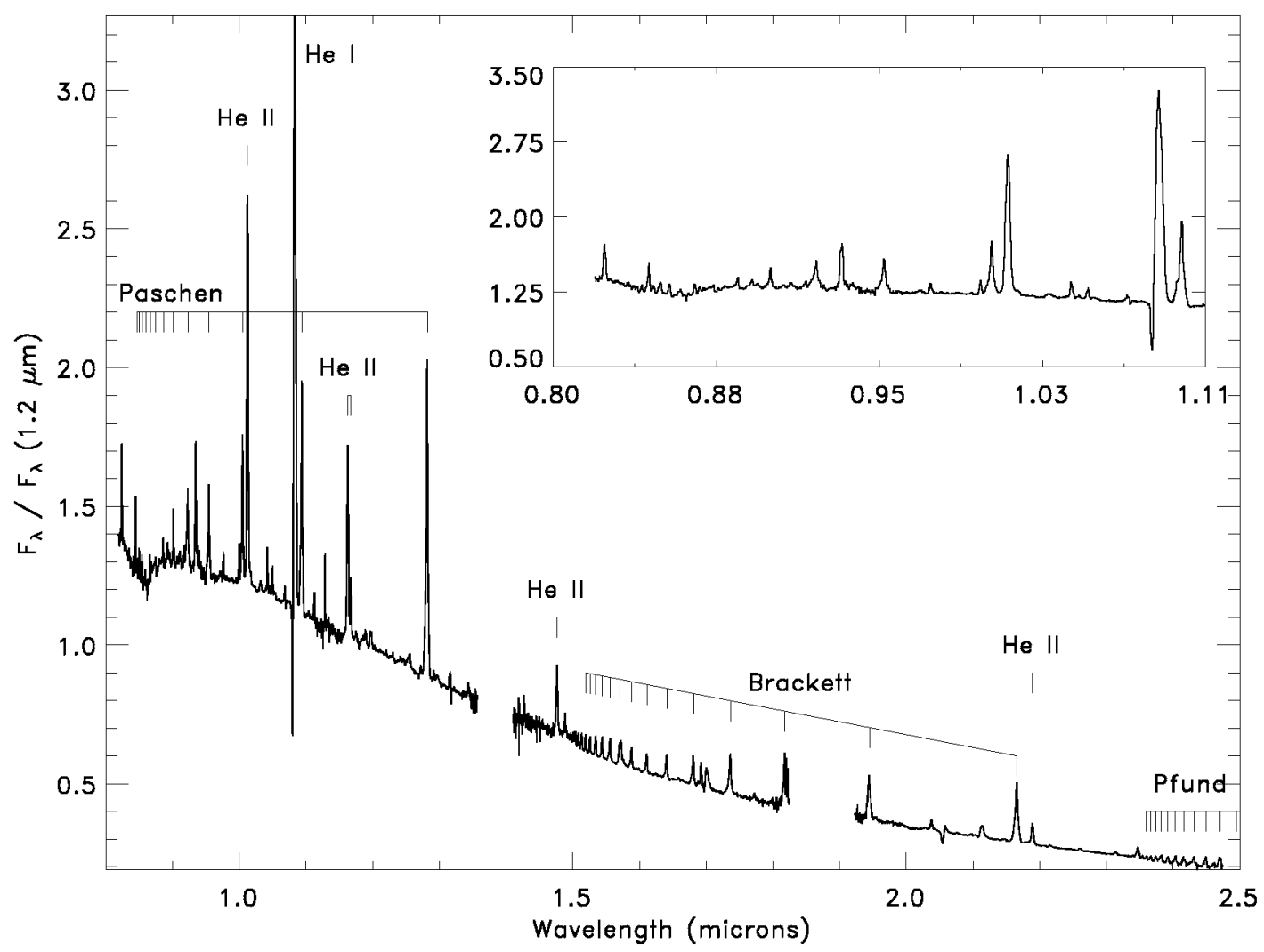

FIG. 10.- $0.8-2.4 \mu \mathrm{m}$ spectrum of the Wolf-Rayet star WR 158 obtained with the SXD mode at $R=2000$. The inset shows detail in the $0.82-1.11 \mu \mathrm{m}$ region including a P Cygni profile in the $\mathrm{He}$ I line at $1.080 \mu \mathrm{m}$.

tion). At $0.4 \mathrm{~V}$ bias, the full-well depth is $111,000 e^{-}$, which is linear to $5 \%$ up to about 52,000 $e^{-}$. An algorithm to correct for nonlinearity is incorporated into Spextool. Cosmetically, the array has two long 1 pixel wide cracks (which have negligible effect on data) and very good uniformity. The operating temperature of $30 \mathrm{~K}$ was found to be the best compromise between dark current, which decreases with temperature, and read noise, which increases with temperature.

A generic problem with Aladdin arrays is residual image, which manifests itself as enhanced dark current when a bright object or high-background exposure is followed by a low-background exposure. Although the blanked-off dark current of the spectrograph array is $0.2 e^{-} \mathrm{s}^{-1}$, the effective dark current during regular observing is up to $1 e^{-} \mathrm{s}^{-1}$ (or a few percent of the previous exposure, whichever is greater) since object integrations are usually bracketed by brighter telluric standardstar integrations and calibrations (flat fields and arcs). We have found that executing global array resets at $1 \mathrm{~Hz}$ when the array is not integrating reduces residual image effects significantly (although still only to the level discussed above). However, since global resets dissipate power locally on the chip, the array cools during long integrations when global resets are stopped, causing the bias level and spatial structure to change during the first integration of a series. Consequently, the first (A beam minus B beam) bias subtraction is not perfect and adds some noise, somewhat offsetting the theoretical gain in $\mathrm{S} / \mathrm{N}$ obtained by integrating longer. To overcome the bias change, we recommend that observers do a $60 \mathrm{~s}$ integration before starting integration sequences with on-chip integration times longer than a few minutes. This "preimage" minimizes the bias change during the actual integration sequence. Also, the preimage is short enough that the global array reset procedure still reduces residual image effects.

The mask developed for PAIDAI contains one $1024 \times$ 1024 die in the center of the InSb wafer, two $1024 \times 512$ dies at the top and bottom, two $512 \times 512$ dies on one side, and five $256 \times 256$ dies on the opposite side. Because of their distance from the center of the wafer, the $512 \times 512$ dies are usually of lower quality than the $1024 \times 1024$ die. We selected the Aladdin $2512 \times 512$ array SCA46509 for use in the slit viewer/guider. At $30 \mathrm{~K}$ and $0.4 \mathrm{~V}$ bias, SCA46509 has a dark current of 1.5 $e^{-} \mathrm{s}^{-1}$, a read noise of $65 e^{-} \mathrm{rms}$ (reset-read-read) measured at a readout rate of $3 \mathrm{~Hz}$, a well depth of $88,000 e^{-}$, which is linear to $10 \%$ up to about $37,000 e^{-}$, and good uniformity.

\subsection{Cryostat Assembly and Testing}

Eight cold tests of the cryostat in the lab were required over the period 1998 September to 2000 April before the instrument 


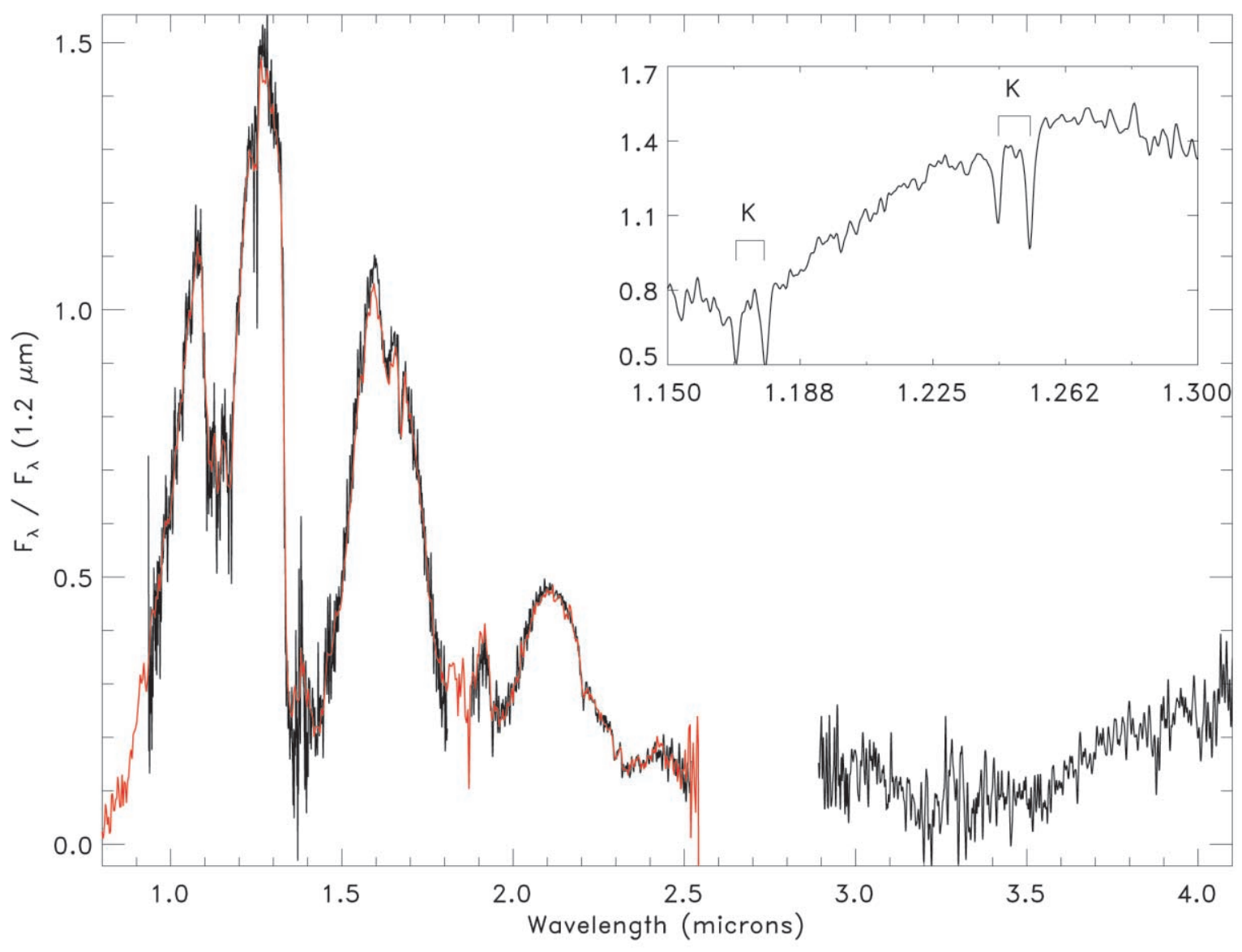

FIG. 11. - 0.9-4.1 $\mu \mathrm{m}$ spectrum of the methane dwarf SDSS 1254-0122 (T2 V) obtained with the SXD mode at $R=1200$ and with the LXD mode at $R=$ 940. A $0.8-2.55 \mu \mathrm{m}$ spectrum obtained with the prism mode at $R \sim 250$ is overplotted (red). The inset shows the $1.15-1.30 \mu \mathrm{m}$ of the SXD spectrum.

was shipped to the telescope for commissioning. In order, these tests verified cryogenic performance, optical alignment and stray light, flexure, and finally all-up instrument performance. The only significant optical adjustments required were positioning of the arrays and alignment of the prisms. Our design philosophy was to build to tolerance and pin all optical assemblies, with some shimming if necessary, rather than build more complex adjustable mounts. The fully assembled cold structure was inspected for light leaks. This was done by placing small xenon flashlights at key locations inside the sealed light path and inspecting the cold structure inside a darkroom using image-intensified goggles. Light leaks were spotted at several locations and fixed. Flexure tests of the instrument were done in all axes on a lift/tilt table. Initially, flexures of up to 10 pixels were measured on the spectrograph array, but these were largely removed by stiffening the springs in the grating turret and slit wheel mechanisms and by balancing the rotation of the grating turret. The final values are 2 pixels in the east, 0.5 pixels in the west, 0.5 pixels in the north, and 0.75 pixels in the south, when tilting to $60^{\circ}$ from vertical. Movements of the reimaged slit were all less than 0.5 pixels.

\section{OPERATION AT THE TELESCOPE}

SpeX was commissioned on IRTF during 2000 May, June, and July and became generally available for Time Allocation Committee awarded telescope time in 2000 August. Since then, SpeX has operated on IRTF for about $50 \%$ of the available time and with very few problems.

\subsection{Observing Procedure}

The general observing procedure is as follows. First, the observer selects the required slit size. The object is then imaged with the slit viewer/guider and placed in the slit. There are several options for guiding. Autoguiding can be done with the infrared slit viewer either on spillover from the object placed in the slit or on an object elsewhere in the $60^{\prime \prime} \times 60^{\prime \prime}$ field of view and at any position angle of the rotator. In the crossdispersed and prism modes, guiding can be done through any 


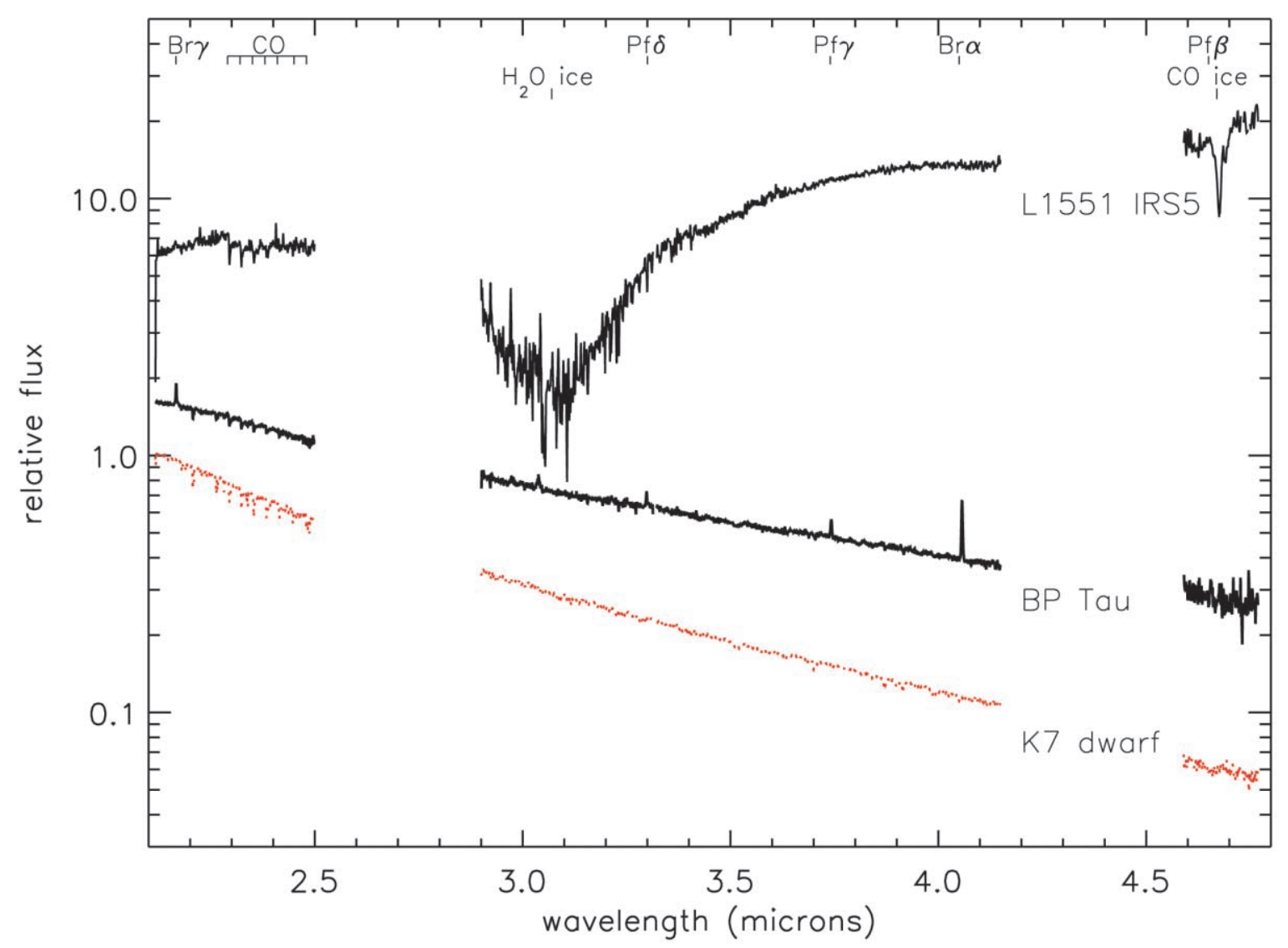

FIG. 12.-2.1-4.8 $\mu \mathrm{m}$ spectra of YSOs obtained with the LXD mode at $R=1500$. L1551 IRS 5 is an embedded protostar, and BP Tau is a classical T Tauri star. A template star of similar spectral type $(\mathrm{K} 5 \mathrm{~V})$ is shown for comparison.

filter in the slit-viewer filter wheel. The magnitude limit for autoguiding on spillover depends on slit width and seeing, but the limit is about $J=15$ in an integration time of $10 \mathrm{~s}$. The limit for manual guiding on spillover is about $J=17$ in $10 \mathrm{~s}$. Alternatively, guiding can be done on-axis in the visible using the tip-tilt wave-front sensor package mounted to the side of SpeX. The beam is reflected into the wave-front sensor by the cold dichroic inside SpeX. The tip-tilt sensor package will be replaced with a wave-front sensor running a 36-element $\mathrm{AO}$ system in 2003. Another option is to use the facility off-axis visible guider.

Once guiding is started and the spectroscopy mode is selected, spectrograph integrations can begin. In the SXD and prism modes, where sky background is low, on-chip integration times up to several hundred seconds can be used for faint sources. (See $\S 8.1$ for how to optimize the array for long onchip integration times.) In the LXD modes, sky brightness limits on-chip exposures to $30 \mathrm{~s}$ at $4 \mu \mathrm{m}$ and $2 \mathrm{~s}$ at $5 \mu \mathrm{m}$. For optimum telluric correction, standards should be taken within about 0.05 air masses of the object. Flat-field and argon arc calibrations are usually obtained at every standard star position even though flexure is very small (typically about 0.2 pixels for 0.1 air mass differences). This is done by executing a calibration macro, which takes about 3 minutes to complete.

Using the slit viewer/guider to obtain infrared images for science is straightforward, and the observing procedure is the same as for other infrared cameras.

\subsection{Example Results}

SpeX is being used for a wide variety of projects. Here we illustrate performance using data taken from an infrared spectral survey of the H-R diagram being conducted by three of us (Rayner, Vacca, \& Cushing) and by observations of young stellar objects (YSOs) by J. Muzerolle et al. (2003, in preparation).

Figure 9 shows a $0.8-2.4 \mu \mathrm{m}$ stellar through substellar temperature sequence. The observations were done in the SXD mode at $R=2000$ and at $R=1200$ for the T dwarfs. On dry Mauna Kea nights, correction through the strong telluric bands at 1.1, 1.4 , and $1.9 \mu \mathrm{m}$ is excellent (e.g., K5 V, M4.5 V, and T5 V). The gap in wavelength coverage at $1.81-1.86 \mu \mathrm{m}$ is necessary to maintain a maximum resolving power of $R=2000$ (see $\S 2.2$ ). The other gaps on some objects are due to regions of 
poor telluric correction that have been removed. Integration times ranged from a few tens of seconds on the brighter dwarfs $(J \sim 5)$ to about $2 \mathrm{hr}$ on the $\mathrm{T} 2 \mathrm{~V}(J=14.88$; Leggett at al. 2000). With the exception of the telluric bands, all the highfrequency structure seen in these spectra is real. See, for example, the many $\mathrm{FeH}$ absorption features identified in the spectra of late-M and L dwarfs by Cushing et al. (2003).

At the top of the temperature sequence, the O9 V (35,900 K) star shows the Paschen line series of atomic hydrogen plus several helium lines. Paschen and Brackett hydrogen lines strengthen through B5 V $(15,400 \mathrm{~K})$ to A2 V $(8800 \mathrm{~K})$ before weakening with decreasing temperature. Refractory metal lines strengthen from $\mathrm{F} 3 \mathrm{~V}(6700 \mathrm{~K})$ to $\mathrm{G} 2 \mathrm{~V}(5800 \mathrm{~K})$ through K5 V $(4300 \mathrm{~K})$ at which temperature carbon monoxide absorption at 2.3-2.4 $\mu \mathrm{m}$ increases. At M4.5 V $(3100 \mathrm{~K})$, refractory metal absorptions weaken as a result of condensation and rain-out, while alkali metal and molecular absorptions (metal hydride, water, and carbon monoxide) begin to strengthen. These trends continue through M8 V $(\sim 2300 \mathrm{~K})$ into the brown dwarf regime, which starts at about $\mathrm{L} 4 \mathrm{~V}$ for field dwarfs and which is represented here by an L5 V ( 1800 K). Note the strong and broad water features at 1.4 and $1.9 \mu \mathrm{m}$. The $\mathrm{L}-\mathrm{T}$ transition region (Leggett et al. 2000) is characterized by the onset of methane absorption at about 1.3, 1.6, and $2.2 \mu \mathrm{m}$. These features are first seen in the sequence shown here at T2 V $(\sim 1300 \mathrm{~K})$ and are very strong in the T5 V $(\sim 1100 \mathrm{~K})$. Further dramatic changes are not expected to occur until water vapor begins to condense at about $400 \mathrm{~K}$. The reflected solar spectrum of Uranus $(60 \mathrm{~K})$ is dominated by strong methane and water absorption.

A $0.8-2.4 \mu \mathrm{m}$ spectrum of the Wolf-Rayet star WR 158 observed in the SXD mode at $R=2000$ is shown in Figure 10. The emission features are due to mass loss in a strong stellar wind. The inset shows detail in the $0.82-1.11 \mu \mathrm{m}$ region including a P Cygni profile in the He I line at $1.080 \mu \mathrm{m}$.

A $0.9-4.1 \mu \mathrm{m}$ spectrum of the methane dwarf SDSS 1254-0122 (T2 V) is shown in Figure 11. The spectrum is the combination of a 1.9-4.2 $\mu \mathrm{m}$ LXD observation taken through the 0.8 wide slit $(R=940)$ and the SXD observation from Figure 9. SDSS $1254-0122$ is very faint at $3-4 \mu \mathrm{m}\left(L^{\prime}=\right.$ 12.12 from Stephens et al. 2001) and required an integration time of about $2 \mathrm{hr}$. However, the fundamental methane absorption at $3.3 \mu \mathrm{m}$ is clearly detected. Overplotted in the figure is a spectrum obtained with the low-resolution prism mode through the 0.3 wide slit $(R \sim 250$; see $\S 2.4)$. The prism spec- trum covers the range $0.8-2.55 \mu \mathrm{m}$ with no gaps. The prism mode is very efficient and overcomes some of the limitations of the SXD mode, where read noise and dark current (enhanced by persistence) of the Aladdin 3 array prevent backgroundlimited performance at $R=2000$. Note the excellent correspondence between the prism and SXD spectrum, confirming that the cross-dispersed orders in the SXD mode are being combined correctly. The inset shows the $1.15-1.30 \mu \mathrm{m}$ region of the SXD spectrum.

Figure 12 shows $2.1-4.8 \mu \mathrm{m}$ spectra of the embedded protostar L1551 and the classical T Tauri star BP Tau compared to a standard star of similar spectral type (K7 V), observed in the LXD mode by J. Muzerolle et al. (2003, in preparation). Note the excellent telluric cancellation. There is clear continuum infrared excess emission arising from accretion disks in both YSOs. In BP Tau, weak photospheric are seen in the $K$ and $L$ bands plus hydrogen emission lines from the accretion disk. In L1551, carbon monoxide absorption at 2.3-2.4 $\mu \mathrm{m}$ is from the disk, and broad water absorption at $3 \mu \mathrm{m}$ and carbon monoxide ice at $4.7 \mu \mathrm{m}$ are probably due to ice-covered grains in the circumstellar envelope.

More details of SpeX can be found at the SpeX Web page. The information includes an $\mathrm{S} / \mathrm{N}$ calculator, a user's manual, and engineering photographs and drawings.

Building SpeX for IRTF has been a team effort. It is a pleasure to thank successive IRTF Division Chiefs Bob Joseph, Tom Greene, and Alan Tokunaga, and instrument technicians Greg Ching and Darryl Watanabe, all kept under control by IRTF project assistant Ms. Karan Hughes. Major parts of SpeX were made in the Institute for Astronomy (IfA), University of Hawaii at Mānoa by machinists Ray Gruber, Sean Kawamoto, Kelly Collins, Randy Chung, and Danny Cook, under the direction of Lou Robertson. At the telescope, SpeX was handled by the IRTF day crew, Paul Jensen, George Koenig, Lars Bergknut, Danley Lee, Imai Namahoe, and Sammie Pung, and observing was assisted by telescope operators Paul Fukumura-Sawada, Bill Golisch, Dave Griep, Charlie Kaminski, and Paul Sears. Money matters were somehow managed by Gale Yamada and Chris Kaukali. Special thanks also to Don Hall for suggesting a medium-resolution cross-dispersed IR spectrograph for IRTF in the first place. Funding for SpeX was provided by the National Science Foundation, NASA, and the University of Hawaii. 


\section{REFERENCES}

Cushing, M. C., Rayner, J. T., Davis, S. P., \& Vacca, W. D. 2003, ApJ, 582, 1066

Fowler, A. M., \& Gatley, I. 1990, ApJ, 353, L33

Greene, T. P, Tokunaga, A. T., Toomey, D. W., \& Carr, J. S. 1993, Proc. SPIE, 1946, 313

Leggett, S. K., et al. 2000, ApJ, 536, L35

Maihara, T., Iwamuro, F., Yamashita, T., Hall, D. N. B., Cowie, L. L., Tokunaga, A. T., \& Pickles, A. 1993, PASP, 105, 940

Schroeder, D. J. 2000, Astronomical Optics (San Diego: Academic Press)
Shure, M. A., Toomey, D. W., Rayner, J. T., Onaka, P. M., \& Denault, A. J. 1994, Proc. SPIE, 2198, 214

Stephens, D. C., Marley, M. S, Noll, K. S., \& Chanover, N. 2001, ApJ, 556, L97

Tokunaga, A. T., Toomey, D. W., \& Carr, J. S. 1990, Proc. SPIE, 1235,131

Vacca, W. D., Cushing, M. C., \& Rayner, J. T. 2003, PASP, in press 